\title{
Article \\ Geometric Justification of the Fundamental Interaction Fields for the Classical Long-Range Forces
}

\author{
Vesselin G. Gueorguiev ${ }^{1,2, *(D)}$ and Andre Maeder ${ }^{3}$ (D) \\ 1 Institute for Advanced Physical Studies, Sofia 1784, Bulgaria \\ 2 Ronin Institute for Independent Scholarship, 127 Haddon Pl., Montclair, NJ 07043, USA \\ 3 Geneva Observatory, University of Geneva, Chemin des Maillettes 51, CH-1290 Sauverny, Switzerland; \\ andre.maeder@unige.ch \\ * Correspondence: Vesselin@MailAPS.org
}

Citation: Gueorguiev, V.G.;

Maeder, A. Geometric Justification of the Fundamental Interaction Fields for the Classical Long-Range Forces. Symmetry 2021, 13, 379. https:// doi.org/10.3390/sym13030379

Academic Editor: Abraham A. Ungar

Received: 5 February 2021

Accepted: 22 February 2021

Published: 26 February 2021

Publisher's Note: MDPI stays neutral with regard to jurisdictional claims in published maps and institutional affiliations.

Copyright: (c) 2021 by the authors. Licensee MDPI, Basel, Switzerland. This article is an open access article distributed under the terms and conditions of the Creative Commons Attribution (CC BY) license (https:// creativecommons.org/licenses/by/ $4.0 /)$.

\begin{abstract}
Based on the principle of reparametrization invariance, the general structure of physically relevant classical matter systems is illuminated within the Lagrangian framework. In a straightforward way, the matter Lagrangian contains background interaction fields, such as a 1-form field analogous to the electromagnetic vector potential and symmetric tensor for gravity. The geometric justification of the interaction field Lagrangians for the electromagnetic and gravitational interactions are emphasized. The generalization to $E$-dimensional extended objects ( $p$-branes) embedded in a bulk space $\mathrm{M}$ is also discussed within the light of some familiar examples. The concept of fictitious accelerations due to un-proper time parametrization is introduced, and its implications are discussed. The framework naturally suggests new classical interaction fields beyond electromagnetism and gravity. The simplest model with such fields is analyzed and its relevance to dark matter and dark energy phenomena on large/cosmological scales is inferred. Unusual pathological behavior in the Newtonian limit is suggested to be a precursor of quantum effects and of inflation-like processes at microscopic scales.
\end{abstract}

Keywords: diffeomorphism invariant systems; reparametrization-invariant matter systems; matter lagrangian; homogeneous singular lagrangians; relativistic particle; string theory; extended objects; p-branes; interaction fields; classical forces beyond electromagnetism and gravity; generally covariant theory; gauge symmetries; background free theories

PACS: 03.50.-z; 04.90.+e; 11.10. Ef; 11. 25.-w; 11.90.+t; 95.35.+d

\section{Introduction}

Probing and understanding physical reality goes through a classical interface that shapes our thoughts as classical causality chains. Therefore, understanding the essential mathematical constructions in classical mechanics and classical field theory is important, even though quantum mechanics and quantum field theory are regarded as more fundamental than their classical counterparts. Two approaches, the Hamiltonian and the Lagrangian, are very useful in theoretical physics [1-6]. In general, there is a transformation that relates these two approaches - the Legendre transform [1-4]. For reparametrizationinvariant models, however, there are problems in changing from Lagrangian to the Hamiltonian approach $[2-5,7,8]$.

Fiber bundles provide the mathematical framework for classical mechanics, field theory, and even quantum mechanics when viewed as a classical field theory. Parallel transport, covariant differentiation, and gauge symmetry are very important structures $[9,10]$ associated with fiber bundles. When asking: "What structures are important to physics?", one should also ask: "Why one fiber bundle should be more "physical' than another?", "Why does the 'physical' base manifold seem to be a four-dimensional Lorentzian manifold?" [11-13], and "How should one construct an action integral for a given fiber bundle?" [1,6,14-16]. Starting 
with the tangent or cotangent bundle seems natural because these bundles are related to the notion of a classical point-like matter. Since knowledge is accrued and tested via experiments that involve classical apparatus, the physically accessible fields should be generated by matter and should couple to matter as well. Therefore, the matter Lagrangian should contain the interaction fields, not their derivatives, with which classical matter interacts [17].

In what follows, the principle of reparametrization invariance is illustrated as a guiding principle in formulating physically relevant models. The symmetry of reparametrization invariance is a common feature of many important physics models but it has been often treated as an issue that needs to be resolved to make reasonable predictions within each specific model. Since any model based on a Lagrangian can be reformulated into an equivalent reparametrization invariant model $[2,18]$, this symmetry may be signaling an important fundamental principle. Thus, focusing the discussion on models with Lagrangians that possess such reparametrization invariance does not restrict the generality of the models considered but instead provides an important classification of the possible physical systems and their interactions. In a nutshell, the principle of reparametrization invariance is like the covariance principle but about the internal coordinates of the physical process under study.

The covariance principle is effectively related to the diffeomorphism symmetry of a manifold $M$. In the theory of manifolds, switching from one chart on $M$ to another is physically equivalent to switching from one observer to another in the spacetime of the observer $M$. Thus, coordinate independence of the physical laws and their mathematical forms when formulated in the manifold framework of $M$. However, the framework does not say anything about a specific physical process $E$ until one makes the relevant manifold model.

A physical process $E$ can be viewed as a manifold that consists of the points involved in the process and their relationships. Thus, processes and their studies can be viewed as the embedding of manifolds. That is, how should $E$ be embedded in $M$ ? For example, the motion of a point particle is just about the trajectory of a particle as viewed as the 1-dimensional curve in the $4 \mathrm{D}$ space-time of the physical observers. Since a process $E$ is also viewed as a manifold then there are mathematical charts that describe $E$ locally. The description of the process (embedding $E \hookrightarrow M$ ) should not depend on the choice made for the charts on $E$. This is an additional symmetry to the covariance principle that things should not depend on the choice of the observer's coordinates for $M$.

Thus, the mathematical framework should also possess diffeomorphisms symmetry of the manifold $E$. That is, in the case of point particle, a 1D curve (the trajectory) mapped into another topologically equivalent 1D curve (but same trajectory) should not change our understanding and the description of the motion of a point particle. Physically, one talks about the covariance principle when considering the diffeomorphisms symmetry of a manifold $M$ and about reparametrization invariance when considering the diffeomorphisms symmetry of the manifold $E$ that is embedded in $M$.

The current research suggests that reparametrization invariance can be achieved by using the Lagrangian formulation with Lagrangians that are homogeneous functions of order one with respect to the velocity. This leads to all the subsequent results that justify only electromagnetic and gravitational classical forces at macroscopic scales which is consistent with experimental observations.

This paper aims to illustrate the possibility that physical reality and observed physical laws are related to a mathematical construction guided by the principle of reparametrization invariance for the embedding of manifolds. This principle suggests geometric justification of the fundamental interaction fields for the classical long-range forces-electromagnetism and gravity, as well as possible new classical fields. Are there observable consequences of such fields on microscopic and/or cosmological scales? Are such fields present in nature? Under what conditions could the relevant reparametrization invariant Lagrangians be reduced or not with such fields to the known Lagrangians that contain only gravitational and electromagnetic fields? These are only a few of the far-reaching questions related to the idea of reparametrization invariance and its correspondence to the observed physical 
laws. One day, hopefully, some of the readers of this paper will be able to address these questions fully and answer them completely.

In brief, the paper starts with the relativistic particle $[7,9,14,19]$ aiming to illustrate the main ideas and their generalization to extended objects ( $p$-branes). In answering the question: "What is the Lagrangian for the classical matter?" the proposed canonical matter Lagrangian naturally contains background interaction fields, such as a 1-form field analogous to the electromagnetic vector potential and symmetric tensor that is usually associated with gravity. The guiding principles needed for the construction of the Lagrangians for the interaction fields are also discussed as an illustration of the uniqueness of the Lagrangians for the electromagnetic and gravitational fields. The authors consider this mathematical framework to be a geometric justification of electromagnetism and gravity. The framework presented here seems to be able to go beyond the Feynman's proof of the Maxwell and Lorentz equations that justify electromagnetism from a few simple fundamental principles $[20,21]$.

In Section 2, the Lagrangian for a relativistic particle is given as an example of a reparametrization-invariant action. Section 3 contains arguments in favor of first-order homogeneous Lagrangians; in Section 3.1 are listed some of the good and lesser properties of such models; in Section 3.2 the canonical form of the first-order homogeneous Lagrangians is justified; and in Section 3.3 the canonical structure of a reparametrization invariant Lagrangian for an extended object (p-brane) embedded in a bulk space $\mathrm{M}$ is shown to lead to some familiar Lagrangians, such as the relativistic point particle in an electromagnetic field, the string theory Lagrangian, and the Dirac-Nambu-Goto Lagrangian. The outlined systems are based on first-order homogeneous Lagrangians in the velocity/generalized velocity to achieve reparametrization invariance along with the usual general covariance. Section 4 discusses the physical implication of such Lagrangians, in particular, in Section 4.1 the possibility of classical forces beyond electromagnetism and gravity is studied for the simplest possible Lagrangian system with symmetric fields $S_{n}$ with $n>2$, while in Section 4.2 the notion of proper time is shown to be mostly related to the gravitational term $(n=2)$ of the matter Lagrangian, while in Section 4.3 the consequence of utilizing un-proper time parametrization of a non-reparametrization-invariant action is illustrated. Section 5 justifies the field Lagrangians relevant for the interaction fields, in particular, the uniqueness of the Lagrangian for electromagnetism, as well as the uniqueness of the Hilbert-Einstein action integral for gravity. The conclusions and discussions are given in Section 6 followed by Section 7, which contains relevant theorems framed as problems and exercises.

\section{The Relativistic Particle Lagrangian}

It is well known that localized particles move with a finite 3D speed. In an extended configuration space (4D space-time), when the time is added as a coordinate $\left(x^{0}=c t\right)$, particles move with a constant 4 -velocity $(v \cdot v=$ constant $)$. The 4 -velocity is constant due to the definition $v^{\mu}=d x^{\mu} / d \tau$ that uses the invariance of the proper-time $(\tau)$ mathematically defined via the symmetric tensor $g_{\mu \nu}\left(d \tau^{2}=g_{\mu v} d x^{\mu} d x^{v}\right)$. Physically the proper-time $(\tau)$ is associated with the passing of time measured by a co-moving clock that is at rest with respect to the particle during its motion. In this case, the action integral for a massive relativistic particle has a nice geometrical meaning: It is the time-elapsed along the particle trajectory [9]:

$$
\begin{gathered}
S_{1}=\int d \tau L_{1}(x, v)=\int d \tau \sqrt{g_{\mu v} v^{\mu} v^{v}}, \\
\sqrt{g_{\mu v} v^{\mu} v^{v}} \rightarrow 1 \Rightarrow S_{1}=\int d \tau .
\end{gathered}
$$

However, for massless particles, such as photons, the length of the 4-velocity is zero $\left(g_{\mu v} v^{\mu} v^{v}=0\right)$. Thus, one has to use a different Lagrangian to avoid problems due 
to division by zero when evaluating the Euler-Lagrange equations. In this case, the appropriate "good" action is [9]:

$$
S_{2}=\int L_{2}(x, v) d \tau=\int g_{\mu v} v^{\mu} v^{v} d \tau
$$

Notice that the Euler-Lagrange equations obtained from $S_{1}$ and $S_{2}$ are equivalent, and both are equivalent to the geodesic equation as well:

$$
\begin{aligned}
\frac{d}{d \tau} \vec{v}=D_{\vec{v}} \vec{v}=v^{\beta} \nabla_{\beta} \vec{v} & =0, \\
v^{\beta}\left(\frac{\partial v^{\alpha}}{\partial x^{\beta}}+\Gamma_{\gamma \beta^{\alpha}}^{\alpha} v^{\gamma}\right. & =0 .
\end{aligned}
$$

In General Relativity (GR), the Levi-Civita connection $\nabla_{\beta}$, with Christoffel symbols $\Gamma_{\beta \gamma}^{\alpha}=g^{\alpha \rho}\left(g_{\rho \beta, \gamma}+g_{\rho \gamma, \beta}-g_{\beta \gamma, \rho}\right) / 2$, preserves the length of the vectors $(\nabla g(\vec{v}, \vec{v})=0)[9]$. Therefore, these equivalences are not surprising because the Lagrangians in (1) and (2) are functions of the preserved arc length $g(\vec{v}, \vec{v})=\vec{v}^{2}$. In principle, however, the parallel transport for an arbitrary connection $\nabla_{\beta}$ does not have to preserve the length of a general vector $[9,10]$. This is clearly seen when metric tensor is velocity dependent and thus the usual argument will not apply [22].

Remarkably, however, going beyond length preserving parallel transport may still hold such equivalence. For example, the Weyl's integrable geometry does have such equivalence between what one expects to be the generalized geodesic equation and the equation derived from an appropriate Lagrangian [23]. Weyl's integrable geometry provides a framework that is likely to be relevant to physics [24]. It is based on the original Weyl's gauge symmetry idea where the length of a vector may depend on the gauge choice as well as upon infinitesimal local displacements. In Weyl's integrable geometry, however, this freedom is constrained to constructions where the length of a vector does not change upon a transport along a closed loop. In such geometry, one finds that only an action that is built upon a co-scalar of order $(-1)$ results in trajectory restricting equations of motion that do correspond to the generalized geodesic equation [23] while any other choices built upon co-scalar length $l$ of order $n \neq-1$ results in the statement that $d l$ is a closed one-form, that is, a perfect deferential $(\mathrm{d}(d l)=0)$. In Weyl's geometry terminology a scalar, vector, and a general tensor object $Y_{\mu \ldots \nu}$ is a co-tensor of order $n$ when $\tilde{Y}_{\mu \ldots \nu}=\beta^{n} Y_{\mu \ldots \nu}$ upon the gauge change of the metric tensor $\tilde{g}_{\mu v}=\beta^{2} g_{\mu \nu}$. Thus, the line element $d \tau$ defined as usual to be $d \tau^{2}=g_{\mu \nu} d x^{\mu} d x^{\nu}$ is a co-scalar of order $(+1)$, then the co-tangent vector with components $v^{\mu}=d x^{\mu} / d \tau$ is seen as a co-vector of order $(-1)$. The mathematical framework developed in [23] practically shows that the only reasonable choice of action for a massive particle, within the Weyl's integrable geometry framework, is given by the action integral (1).

The equivalence between $S_{1}$ and $S_{2}$ is very robust. Since $L_{2}$ is a homogeneous function of order 2 with respect to $\vec{v}$, the corresponding Hamiltonian function $(h=v \partial L / \partial v-L)$ is equal to its Lagrangian $\left(h(x, v)=L_{2}(x, v)\right)$. As long as there is no explicit proper-time dependence then $L_{2}$ is conserved, and so is the length of $\vec{v}$. Any parameter independent homogeneous Lagrangian in $\vec{v}\left(L_{n}(x, \beta v)=\beta^{n} L_{n}(x, v)\right)$ of order $n \neq 1$ is conserved because $h=(n-1) L_{n}$. When $d L / d \tau=0$, then one can show that the Euler-Lagrange equations for $L$ and $\tilde{L}=f(L)$ are equivalent under certain minor restrictions on $f$ (see Section 4.3 for more details). This is an interesting type of equivalence that applies to homogeneous Lagrangians. It is different from the usual equivalence $L \rightarrow \tilde{L}=L+d \Lambda / d \tau$ or the more general equivalence discussed in [25]. Any solution of the Euler-Lagrange equation for $\tilde{L}=L^{\alpha}, \alpha \neq 1$ would conserve $L=L_{1}$ since $\tilde{h}=(\alpha-1) L^{\alpha}$ is conserved. All these solutions are solutions of the Euler-Lagrange equation for $L$ as well; thus $L^{\alpha} \subset L$ in the sense of their set of solutions. In general, conservation of $L_{1}$ is not guaranteed since $L_{1} \rightarrow L_{1}+d \Lambda / d \tau$ is also a first-order homogeneous Lagrangian in the velocities that is equivalent to $L_{1}$. This suggests that there could be a choice of $\Lambda$, a "gauge fixing", such that $L_{1}+d \Lambda / d \tau$ is conserved even if $L_{1}$ is not. However, whenever $L_{1}$ is conserved, then the corresponding 
equations would also be related to the geodesic Equation (3) as well. Relevant examples will be discussed in the next paragraphs but before doing so, hands-on readers may benefit more if they do the first four problems of the exercises in Section 7.

The simplest example is the case $L_{1}(x, v)=m \sqrt{g_{\mu v} v^{\mu} v^{v}}$ and $L_{2}(x, v)=\frac{m}{2} g_{\mu v} v^{\mu} v^{v}$. Notice that the details of the mass multiplier are actually irrelevant in this case since the corresponding Euler-Lagrange Equation (4) is insensitive to its value. The mass $m$, however, comes into the picture as integral of the motion as soon as we consider the relevant energy-momentum dispersion relation $p_{v} p^{v}=m^{2}$. For $L_{2}$ the geometric linear momentum $\left(p_{v}=m g_{\mu \nu} v^{\mu}\right)$ and the generalized linear momentum $\left(\pi_{\mu}=\partial L_{2} / \partial v^{\mu}\right)$ coincide. While in the case of $L_{1}$, the generalized linear momentum $\pi_{\mu}^{\prime}=\partial L_{1} / \partial v^{\mu}=\frac{m g_{\mu v} v^{\mu}}{\sqrt{g_{\mu v} v^{\mu} v^{v}}}$ differs from the geometric one by a factor $\frac{m}{L_{1}}=1 / \sqrt{g_{\mu \nu} v^{\mu} v^{v}}$. The two linear momenta can be made the same if this factor is forced to be equal to one, that is, to use the usual choice of proper-time parametrization that results in $g_{\mu v} v^{\mu} v^{v}=1$. This however, is only possible for massive particle $m \neq 0$ while for massless particles it is clearly a contradiction with their null-geodesic equation $g_{\mu \nu} v^{\mu} v^{v}=0$. Thus, for $m \neq 0$ the two Lagrangians are equivalent as long as one recognizes the use of the proper-time parametrization. However, there is a slight nuance here, based on the reparametrization invariance of $S_{1}$ one can see the choice of proper-time parametrization as a matter of convenience that results in $g_{\mu \nu} v^{\mu} v^{v}=1$, while for $L_{2}$ this is a matter of "physics" content since $S_{2}$ does not possess reparametrization invariance. Thus, a "clever" choice of parametrization that reflects the physical reality has to be imposed. After all, it is still the same proper-time parametrization but it is justified after looking at the Hamiltonian function for $L_{2}$ and recognizing that such choice of parametrization would show explicitly that the Hamiltonian function corresponds to an integral of motion. If one uses arbitrary un-proper parametrization then one is likely to come across factious acceleration as discussed in Section 4.3.

As stated already, the mass multiplier is irrelevant as seen from the corresponding Euler-Lagrange Equation (4) and therefore one can consider $S_{2}$ only with $L_{2}(x, v)=$ $g_{\mu v} v^{\mu} v^{v}$ and use $p_{v} p^{v}=m^{2}$ as a way of assessing the mass of a particle. This is particularly useful for particles that follow the null-geodesic equation $g_{\mu v} v^{\mu} v^{v}=0$ while the treatment of $S_{1}$ is more complicated [9]. The problem with $S_{1}$ steams from the fact that now the generalized momentum $\pi_{\mu}^{\prime}$ is ill defined and cannot be easily made equal to the geometric momentum $p_{\mu}$. Nevertheless, if one keeps track of the factor $\sqrt{g_{\mu \nu} v^{\mu} v^{v}}$ when analyzing $S_{1}$ one can see that the corresponding Euler-Lagrange equations are the same as those for $S_{2}$ if one imposes the condition $g_{\mu v} v^{\mu} v^{v}=$ constant. Thus, in this example $S_{1}$ and $S_{2}$ are equivalent as long as $g_{\mu \nu} v^{\mu} v^{v}$ is an integral of the motion. This condition is easily seen to be valid for $S_{2}$ due to the fact that the Hamiltonian function for $L_{2}$ is equal to $L_{2}$. Thus any solution related to $L_{2}$ will correspond to a solution for $L_{1}$ that satisfies, in this case a supplemental condition, $g_{\mu v} v^{\mu} v^{v}=$ constant. Therefore, solutions for $L_{1}$ that do not satisfy this "physical" condition will be un-physical since they will not be solutions related to $L_{2}$. In this respect $L_{2} \subset L_{1}$.

The "physical" assumption $g_{\mu v} v^{\mu} v^{v}=$ constant is a key ingredient of the parallel transport considerations in Einstein General Relativity (GR), which seems to be obeyed by nature. After all, studying processes out there in the universe, especially these that are particularly far from our labs, can be easily understood if this condition was satisfied and thus resulting in the corresponding geodesic Equation (3). However, the past few decades of studies on far away galaxies and the universe as a whole have brought some puzzling results that have been attributed to dark matter and dark energy phenomena that have not be confirmed in our local laboratories. It is often commonly expected that dark matter and dark energy are probably a new kind of particles and/or fields that have not yet been experimentally discovered, but perhaps various upcoming efforts [26] to address current discordances present between the different cosmological probes could result in the detection of new interaction fields that may even be relevant to phenomenon of inflation. Very recent research results, literally just submitted for publication by the authors, in effort to understand the Scale Invariant Vacuum (SIV) theory [24,27-29] and its limitations has 
resulted in encouraging connections with standard models of inflation [30]. Interestingly, these dark phenomena may be illuminated and could be understood quite well within the Integrable Weyl geometry paradigm that deviates from the standard Einstein GR parallel transport considerations $[27,29]$. The Integrable Weyl geometry does allow for departing from $g_{\mu v} v^{\mu} v^{v}=$ constant. Thus, it provides a framework with a larger set of solutions, those solutions to $L_{1}$ that are not part of the $L_{2}$ space of solutions, to be explored for a better understanding of nature.

In the above example, the mass multiplier $m$ in the Lagrangian was not relevant for the equations of motion (4) but the mass was showing up as an integral of the motion via the relevant energy-momentum dispersion relation $p_{v} p^{v}=m^{2}$. This is usually the rest-mass of a particle. In general, however, when there are additional interactions, say electromagnetic, then the mass term in the Lagrangian is playing the role of a coupling constant to the gravitational field but it can also manifest itself as a Lagrange multiplier.

Indeed, if one starts with the re-parametrization invariant Lagrangian $L=q A_{\alpha} v^{\alpha}+$ $m \sqrt{g_{\alpha \beta}(x) v^{\alpha} v^{\beta}}$, which is usually interpreted as the relativistic Lagrangian of a massive particle due to the mass parameter $m$. The mass here is actually a coupling constant to the gravitational field $g_{\alpha \beta}$, just like the charge $q$ is playing the role of coupling constant to the electromagnetic vector potential $A_{\alpha}$. By utilizing the reparametrization invariance and defining the proper time $\tau$ such that: $d \tau=\sqrt{g_{\alpha \beta} d x^{\alpha} d x^{\beta}} \Rightarrow \sqrt{g_{\alpha \beta} v^{\alpha} v^{\beta}}=1$, then one can effectively consider $L=q A_{\alpha} v^{\alpha}+(m+\chi) \sqrt{g_{\alpha \beta} v^{\alpha} v^{\beta}}-\chi$ as our model Lagrangian. Here $\chi$ is a Lagrange multiplier to enforce $\sqrt{g_{\alpha \beta} v^{\alpha} v^{\beta}}=1$ that breaks the reparametrization invariance explicitly. Then one can write it as $L=q A_{\alpha} v^{\alpha}+(m+\chi) \frac{g_{\alpha \beta} v^{\alpha} v^{\beta}}{\sqrt{g_{\alpha \beta} v^{\alpha} v^{\beta}}}-\chi$ and using $\sqrt{g_{\alpha \beta} v^{\alpha} v^{\beta}}=1$ one arrives at $L=q A_{\alpha} v^{\alpha}+(m+\chi) g_{\alpha \beta} v^{\alpha} v^{\beta}-\chi$. One can deduce a specific value for $\chi(\chi=-m / 2)$ by requiring that $L=q A_{\alpha} v^{\alpha}+m \sqrt{g_{\alpha \beta}(x) v^{\alpha} v^{\beta}}$ and $L=q A_{\alpha} v^{\alpha}+(m+\chi) g_{\alpha \beta} v^{\alpha} v^{\beta}-\chi$ produce the same Euler-Lagrange equations under the constraint $\sqrt{g_{\alpha \beta} v^{\alpha} v^{\beta}}=1$. Then, by dropping the overall constant term, this finally results in the familiar equivalent Lagrangian: $L=q A_{\alpha} v^{\alpha}+\frac{m}{2} g_{\alpha \beta} v^{\alpha} v^{\beta}$ where $\tau$ has the usual meaning of proper-time parametrization such that $\sqrt{g_{\alpha \beta} v^{\alpha} v^{\beta}}=1$. This quadratic Lagrangian is often considered as more convenient to work with [9] due to a variety of unpleasant properties of the original re-parametrization invariant Lagrangian. In particular, Lagrangians quadratic in the velocity are preferred since the corresponding Hamiltonian function is quadratic in the momentum and apparently is non-zero. However, if one is to keep track of all the manipulations above, then one would notice that the apparently quadratic Hamiltonian $H=\frac{m}{2} g_{\alpha \beta} v^{\alpha} v^{\beta}+\chi$ is identically zero when utilizing $g_{\alpha \beta} v^{\alpha} v^{\beta}=1$ and $\chi=-m / 2$. The next section is devoted to the justification of why the original re-parametrization invariant Lagrangian may be more relevant for understanding the physical reality despite its unpleasant properties. The importance of such re-parametrization invariant Lagrangian as a way of adding metric structure to an affine space has been emphasized previously by Randers in his paper "On an Asymmetrical Metric in the Four-Space of General Relativity" along the various connections to the treatment of gravity and electromagnetism within a similar framework [22].

\section{Homogeneous Lagrangians}

Suppose one does not know classical physics, which is mainly concerned with trajectories of point particles in some space $M$ but is told that can derive it from a variational principle if the right action integral $S=\int L d \tau$ is used. By following the above example, one would wonder: "Should the smallest 'time distance' be the guiding principle?" when constructing $L$. If yes, "How should it be defined for other field theory models?" It seems 
that a reparametrization-invariant theory can provide us with a metric-like structure [7,22], and thus, a possible link between field models and geometric models [31].

In the example of the relativistic particle (Section 2 above), the Lagrangian and the trajectory parameterization have a geometrical meaning. In general, however, parameterization of a trajectory is quite arbitrary for any observer. If there is the smallest time interval that sets space-time scale, then this would imply a discrete space-time structure since there may not be any events in the smallest time interval. The Planck scale is often considered to be such a special scale [32]. Leaving aside recent hints for quantum spacetime from loop quantum gravity and other theories, one should ask: "Should there be any preferred trajectory parameterization in a smooth $4 \mathrm{D}$ space-time?" and "Are we not free to choose the standard of distance (time, using natural units $c=1$ )?" If so, then one should have a smooth continuous manifold and our theory should not depend on the choice of parameterization.

If one examines the Euler-Lagrange equations carefully:

$$
\frac{d}{d \tau}\left(\frac{\partial L}{\partial v^{\alpha}}\right)=\frac{\partial L}{\partial x^{\alpha}}
$$

one would notice that any homogeneous Lagrangian of order $n\left(L(x, \alpha \vec{v})=\alpha^{n} L(x, \vec{v})\right)$ provides a reparametrization invariance of the equations under the transformations $\tau \rightarrow$ $\tau / \alpha, \vec{v} \rightarrow \alpha \vec{v}$. As a side remark, notice that for homogeneous Lagrangian in $x$, the EulerLagrange equations possess scale invariance upon rescaling of the coordinates $x$. In general, such symmetries are related to the freedom of choosing a system of units by the laboratory observer. However, the symmetry is often broken due to the natural scales relevant to the specific process under study. Next, note that the action integral $S$ involves an integration that is a natural structure for orientable manifolds $(M)$ with an $n$-form of the volume. Since a trajectory is a one-dimensional object, then what one is looking at is an embedding:

$$
\phi: \mathbb{R}^{1} \rightarrow M
$$

This means that the map $\phi$ pushes forward the tangential space $\phi_{*}: T\left(\mathbb{R}^{1}\right)=\mathbb{R}^{1} \rightarrow$ $T(M)$, and pulls back the cotangent space $\phi^{*}: T^{*}\left(\mathbb{R}^{1}\right)=\mathbb{R}^{1} \leftarrow T^{*}(M)$. Thus, a 1-form $\omega$ on $M$ that is in $T^{*}(M)\left(\omega=A_{\mu}(x) d x^{\mu}\right)$ will be pulled back on $\mathbb{R}^{1}\left(\phi^{*}(\omega)\right)$ and there it should be proportional to the volume form on $\mathbb{R}^{1}\left(\phi^{*}(\omega)=A_{\mu}(x)\left(d x^{\mu} / d \tau\right) d \tau \sim d \tau\right)$, allowing one to integrate $\int \phi^{*}(\omega)$ :

$$
\int \phi^{*}(\omega)=\int L d \tau=\int A_{\mu}(x) v^{\mu} d \tau .
$$

Therefore, by selecting a 1-form $\omega=A_{\mu}(x) d x^{\mu}$ on $M$ and using $L=A_{\mu}(x) v^{\mu}$ one is actually solving for the embedding $\phi: \mathbb{R}^{1} \rightarrow M$ using a chart on $M$ with coordinates $x: M \rightarrow \mathbb{R}^{n}$.

The Lagrangian obtained this way is first-order homogeneous in the velocity $v$ with very simple dynamics. The corresponding Euler-Lagrange equation is $F_{v \mu} v^{\mu}=0$ where $F$ is a 2 -form $(F=d A)$; in electrodynamics, this is the Faraday's tensor. If one relaxes the assumption that $L$ is a pulled back 1-form and assume that it is just a homogeneous Lagrangian of order one, then one finds a reparametrization-invariant theory that has an important physics-related dynamics.

\subsection{Pros and Cons of Homogeneous Lagrangians of First Order}

Although most of the features listed below are more or less self-evident, it is important to compile a list of properties of the first-order homogeneous Lagrangians in the velocity $\vec{v}$.

Some of the good properties of a theory with a first-order homogeneous Lagrangian are:

(1) First of all, the action $S=\int L\left(x, \frac{d x}{d \tau}\right) d \tau$ is a reparametrization invariant. Thus, there is no fictitious acceleration due to un-proper time parametrization (see Section 4.3); 
(2) For any Lagrangian $L\left(t, x^{i}, \frac{d x^{i}}{d t}\right)$ one can construct a reparametrization-invariant Lagrangian by enlarging the space from $x^{i}: i=1, \ldots, n$ to an extended space-time $x^{\mu}: \mu=0,1, \ldots, n, x^{0}=t[2,18]: L\left(t, x^{i}, \frac{d x^{i}}{d t}\right) \rightarrow L\left(x^{\mu}, \frac{d x^{\mu}}{d x^{0}}\right) \frac{d x^{0}}{d \tau}$. The Euler-Lagrange equations for these two Lagrangians are equivalent as long as $v^{0}=d t / d \tau$ is well behaved and $\tau$ is also a reasonable "time"-parametrization choice;

(3) Parameterization-independent path-integral quantization is possible since the action $S$ is reparametrization invariant;

(4) The reparametrization invariance may help in dealing with singularities [33];

(5) It is easily generalized to extended objects ( $p$-branes) that is the subject of Section 3.3.

The list of trouble-making properties in a theory with a first-order homogeneous Lagrangian includes:

(1) There are constraints among the Euler-Lagrange equations [2] since $\operatorname{det}\left(\frac{\partial^{2} L}{\partial v^{\alpha} \partial v^{\beta}}\right)=0$;

(2) It follows that the Legendre transformation $\left(T(M) \leftrightarrow T^{*}(M)\right.$ ), which exchanges velocity and momentum coordinates $(x, v) \leftrightarrow(x, p)$, is problematic [4];

(3) There is a problem with the canonical quantization approach since the Hamiltonian function is identically ZERO $(h \equiv 0)$ [5].

Constraints among the equations of motion are not an insurmountable problem since there are procedures for quantizing such theories [5,34-37]. For example, instead of using $h \equiv 0$ one can use some of the constraint equations available, or a conserved quantity, as Hamiltonian for the quantization procedure [5]. Changing coordinates $(x, v) \leftrightarrow(x, p)$ seems to be difficult but it may be resolved in some special cases by using the assumption that a gauge $\Lambda$ has been chosen so that $L \rightarrow L+\frac{d \Lambda}{d \tau}=\tilde{L}=$ const. The above-mentioned quantization difficulties would not be discussed since they are outside of the scope of this paper. A new approach that turns the problem $h \equiv 0$ into a virtue and naturally leads to a Dirac-like equation is under investigation and the subject of a forthcoming paper, for some preliminary details see [38,39]. Currently the new quantization approach has resulted in interesting connections and new view points at some of the key properties of physical systems $[18,40]$. Even though the connection to quantum physics is beyond the scope of the current paper, we would like to point out that the results in $[18,40]$ concerned with the consistent quantization of re-parametrization invariance systems, provide a new viewpoint on the choice of the Hamiltonian constraint and the meaning of process parametrization within a chosen quantization frame. In particular, it is shown that the positivity of the rest energy is related to the requirement of normalizability of the states by utilizing the Hamiltonian constraint into a quantum constrain $(\hat{H} \Psi=0)$. In this respect, the results in $[18,40]$ are reproducing the familiar quantum results where the constraint eliminates the ghost states and the ordinary mass shell constraint is related to the Klein-Gordon equation [41,42].

\subsection{Canonical Form of the First-Order Homogeneous Lagrangians}

Hopefully, by now the reader is puzzled, and is wondering along the following line of thinking: "What is the general mathematical expression for first-order homogeneous functions?" In this section, the notion of the canonical form of the first-order homogeneous Lagrangian and why such a form may be a useful mathematical expression from a physics point of view is justified.

First, note that any symmetric tensor of rank $n\left(S_{\alpha_{1} \alpha_{2} \ldots \alpha_{n}}=S_{\left[\alpha_{1} \alpha_{2} \ldots \alpha_{n}\right]}\right.$, where $\left[\alpha_{1} \alpha_{2} \ldots \alpha_{n}\right]$ is an arbitrary permutation of the indexes) defines a homogeneous function of order $n$ $\left(S_{n}(\vec{v}, \ldots, \vec{v})=S_{\alpha_{1} \alpha_{2} \ldots \alpha_{n}} v^{\alpha_{1}} \ldots v^{\alpha_{n}}\right)$ in the velocity $v$. The symmetric tensor of rank two is denoted by $g_{\alpha \beta}$. Using this notation, the canonical form of the first-order homogeneous Lagrangian is defined as:

$$
\begin{aligned}
L(\vec{x}, \vec{v}) & =\sum_{n=1}^{\infty} \sqrt[n]{S_{n}(\vec{v}, \ldots, \vec{v})}= \\
& =A_{\alpha} v^{\alpha}+\sqrt{g_{\alpha \beta} v^{\alpha} v^{\beta}}+\ldots+\sqrt[m]{S_{m}(\vec{v}, \ldots, \vec{v})}
\end{aligned}
$$


Whatever is the Lagrangian for the matter, it should involve interaction fields that couple with the velocity $\vec{v}$ to a scalar. Thus, the matter Lagrangian $L_{\text {matter }}(\vec{x}, \vec{v} ;$ Fields $\Psi)$ would depend also on the interaction fields. When the matter action is combined with the action $\left(\int \mathcal{L}[\Psi] d V\right)$ for the interaction fields $\Psi$, then one obtains a full background independent theory. Then, the corresponding Euler-Lagrange equations contain "dynamical derivatives" on the left-hand side and sources on the right-hand side:

$$
\partial_{\gamma}\left(\frac{\delta \mathcal{L}}{\delta\left(\partial_{\gamma} \Psi\right)}\right)=\frac{\delta \mathcal{L}}{\delta \Psi}+\frac{\partial L_{\text {matter }}}{\partial \Psi}
$$

There are many ways to write first-order homogeneous functions [7]. For example, one can consider the following expression $L(\vec{x}, \vec{v})=\left(h_{\alpha \beta} v^{\alpha} v^{\beta}\right)\left(g_{\alpha \beta} v^{\alpha} v^{\beta}\right)^{-1 / 2}$ where $h$ and $g$ are seemingly different symmetric tensors. However, each one of these fields ( $h$ and $g$ ) has the same source type $\left(\sim v^{\alpha} v^{\beta}\right)$ :

$$
\frac{\partial L}{\partial h_{\alpha \beta}}=\frac{L(\vec{x}, \vec{v})}{h_{\gamma \rho} v^{\gamma} v^{\rho}} v^{\alpha} v^{\beta}, \quad \frac{\partial L}{\partial g_{\alpha \beta}}=\frac{L(\vec{x}, \vec{v})}{g_{\gamma \rho} v^{\gamma} v^{\rho}} v^{\alpha} v^{\beta} .
$$

Theories with two metrics have been studied before $[43,44]$. However, at this stage of our discussion, it seems unclear why the same source type should produce different interaction fields.

Some other relevant examples come from the field of Finsler spacetime geometry and its applications to physics $[45,46]$, in particular the work of Bogoslovsky seems to be culminating in an experimentally testable framework $[47,48]$. In his approach, Bogoslovsky is utilizing a Lagrangian $\sim f_{0}(\vec{n}, \vec{v})^{b} \sqrt{1-v^{2} / c^{2}}$ that has an explicit isotropy breaking effect $\vec{n}$ due to a conformal factor $f_{0}(\vec{n}, \vec{v})$ based on zeroth-order homogeneous function $f_{0}$ in the velocity of a particle $\vec{v}$. The power $b$ is a parameter that at $b=0$ results in the usual "gravity"-like interaction while when $b=1$, then the system seems to be more involved in an "electromagnetic"-like interaction. Using a standard gravity-like interaction along with a zeroth-order homogeneous function is an alternative mathematical approach, $L=f_{0}(x, v) \sqrt{g_{\mu v} v^{\mu} v^{v}}$, since one expects gravity to always be present. Furthermore, such functional form for $L(x, v)$ has a good justification based on the Ehlers-Pirani-Schild axiomatic approach to Finsler geometry along with some additional requirements on the functional form of $f_{0}$ [46]. Unfortunately, besides the Bogoslovsky case where the isometry breaking field $\vec{n}$ and its consequences are well studied, the approach based on $L=f_{0}(x, v) \sqrt{g_{\mu v} v^{\mu} v^{v}}$ is not yet helpful in understanding the general structure of $f_{0}$, the meaning of the interaction fields, which will be involved in its content, and their classification. Even more, it is not clear if electromagnetic phenomenon could be described appropriately with such particular Lagrangian.

The advantage of the canonical form of the first-order homogeneous Lagrangian (6) is that each interaction field, which is associated with a symmetric tensor, has a unique matter source that is a monomial in the velocities:

$$
\frac{\partial L}{\partial S_{\alpha_{1} \alpha_{2} \ldots \alpha_{n}}}=\frac{1}{n}\left(S_{n}(\vec{v}, \ldots, \vec{v})\right)^{\frac{1-n}{n}} v^{\alpha_{1}} \ldots v^{\alpha_{n}} .
$$

Therefore, the canonical form (6) is a natural choice for further discussion of the firstorder homogeneous Lagrangians. Moreover, if one embraces the principle of one-to-one correspondence between an interaction field and its source, then the canonical form of the first-order homogeneous Lagrangian (6) via (7) justifies, from the mathematical point of view, the presence of the electromagnetic and gravitational fields in nature.

If one could devise a unique procedure to express any first-order homogeneous function in the canonical form above by using only the first two terms, then this could be viewed as a mathematical explanation of the unique physical reality of only two fundamental classical interactions-the electromagnetic and gravitational interactions. The first suggestion for such a procedure is given in problem 11 of the exercises in Section 7 . When 
applied to a Lagrangians that contain only electromagnetism and gravity only $(n=1 \& 2)$, then the procedure recovers the original Lagrangian. For other Lagrangians it can produce effective electromagnetism and gravity-only Lagrangian. However, the equivalence or the specific accuracy of the approximation to the original equations of motion is not yet clear.

Following the Randers and Finsler path one could formally split a general reparametrization invariant model based on first-order homogeneous Lagrangian $L(x, v)$ into electromagnetic and gravitational-like interactions as an even and odd part of $L(x, v)=$ $L^{(-)}(x, v)+L^{(+)}(x, v)$. Where $L^{( \pm)}(x, v)=(L(x, v) \pm L(x,-v)) / 2$. Electromagnetic effects are then related to $q A_{\mu}(x, \vec{v} / c):=\partial L^{(-)}(x, v) / \partial v^{\mu}$ where the electromagnetic (E\&M) four-vector potential has velocity dependence only on the special 3D velocity $\vec{v}=d \vec{x} / d t$; thus, it is homogeneous function of order zero. Example of such velocity dependent E\&M fields and its importance has been discussed by Carlip [49]. Next the gravitational effects could now be related to the even part of the Lagrangian by considering the corresponding Finslerian metric $m^{2} g_{\mu v}(x, v):=\partial^{2} L^{(+)}(x, v)^{2} / \partial v^{\mu} \partial v^{v}$ that is expecting to be a homogeneous function of order zero in the velocity and thus to have dependence only on the special $3 \mathrm{D}$ velocity $\vec{v}=d \vec{x} / d t$ as well. Again the velocity dependent gravitational potentials are essential to the understanding of the relation to retarded potentials and so on [49]. This is a simple justification of why there are only gravity and electromagnetism on a classical level where the test particles are probing the fields far from their sources. However, there are further details to be worked out, like the equations satisfied by these classical fields, and as to what level this procedure actually reproduces the original Lagrangian i.e., see exercise 11. The answers to all these questions however depend on the specific Lagrangian to be studied. An alternative is to study the canonical form (6) were the fields are velocity independent.

Thus, it is important to investigate the additional higher-order terms and their relevance to our physical reality. However, before entering into such a discussion, which is the focus of Section 4, it will be interesting to touch upon the geometric description behind the principle of reparametrization invariance. In this respect, the next subsection will discuss examples of the relevant Lagrangians that provide an illustration of the power of that principle in the justification of important Lagrangian-based models such as relativistic point particle, strings, and $p$-branes in theoretical and mathematical physics.

\subsection{E-dimensional Extended Objects}

At the beginning of the current section, the classical mechanics of a point-like particle has been discussed as a mathematical problem concerned with the embedding $\phi: \mathbb{R}^{1} \rightarrow M$ (5). The map $\phi$ provides the description of the trajectory (the word line) of the particle in the target space $M$. The actual coordinate realization of the map $\phi$ depends on the choice of the Lagrangian $L$ and the interaction fields in $M$ due to the other objects that are already in $M$. According to the canonical form of the first-order homogeneous Lagrangians (6), a point particle would interact with electromagnetic-like vector field $A_{\mu}(x)$ and gravitation-like (symmetric rank 2 tensor) field $g_{\mu \nu}(x)$, as well as with other possible classical long-range fields (7) that are described via rank $n>2$ symmetric tensors $S_{\alpha_{1} \alpha_{2} \ldots \alpha_{n}}(x)$.

These interaction fields can be viewed as an embedding of higher-dimensional objects. For example, $A_{\mu}(x)$ may be viewed as an embedding of $M$ into space with the same dimension $m$, for electromagnetism, it is $4 D$ space into another $4 D$ space. For gravity, it is about $4 D$ space into a $10 D$ space since there are 10 independent entries in a symmetric $4 \times 4$ matrix $g_{\mu v}(x)$. However, one does not have to consider only the interaction fields for a point particle. One can consider a more general extended object called $p$-brane. In this sense, the classical mechanics of a point-like particle that has been discussed as a problem concerned with the embedding $\phi: \mathbb{R}^{1} \rightarrow M$ is actually a 0-brane that is a one-dimensional object. Although time is kept in mind as an extra dimension, one should not insist on any special structure associated with a time flow. For this reason there will be no 0-label(s), which usually singles out the time component(s), in this section.

Let us think of an extended object as a manifold $E$ with dimension, denoted also by $E, \operatorname{dim} E=E=p+1$ where $p=0,1,2, \ldots$ In this respect, one has to solve for $\phi: E \rightarrow M$ 
such that some action integral is minimized. From this point of view, one is dealing with the mechanics of a $p$-brane. In other words, how is this E-dimensional extended object, which is representing the "trajectory" of a $p$-brane, submerged in $M$, and what are the relevant interaction fields? By using the coordinate charts on $M\left(x: M \rightarrow \mathbb{R}^{m}\right)$, one can think of this as a field theory over the $E$-manifold with a local fiber $\mathbb{R}^{m}$. Thus the field $\vec{\phi}$ is such that:

$$
\vec{\phi}: \phi^{\alpha}=x^{\alpha} \circ \phi: E \rightarrow M \rightarrow \mathbb{R}^{m} .
$$

Following the relativistic point particle discussion after Equation (5), but this time using the pullback $\phi^{*}$ of the embedding map $\phi$ in (8), one considers the space of the $E$-forms over the manifold $M$, denoted by $\Lambda^{E}(M)$ with dimension $D=\left(\begin{array}{c}m \\ E\end{array}\right)=\frac{m !}{E !(m-E) !}$. In a specific coordinate basis a general element $\Omega$ in $\Lambda^{E}(M)$ has the form $\Omega=\Omega_{\alpha_{1} \ldots \alpha_{E}} d x^{\alpha_{1}} \wedge d x^{\alpha_{2}} \wedge$ $\cdots \wedge d x^{\alpha_{E}}$. Since $m \geq E$ for such an embedding then there are $D$ linearly independent $E$ forms in $\Lambda^{E}(M)$. Let us use an arbitrary label $\Gamma=1,2, \ldots, D$ to index the different $E$-forms over $M$; thus, $\Omega^{\Gamma}=\Omega_{\alpha_{1} \ldots \alpha_{E}}^{\Gamma} d x^{\alpha_{1}} \wedge d x^{\alpha_{2}} \wedge \cdots \wedge d x^{\alpha_{E}}$ are total of $D$ linearly independent E-forms in $\Lambda^{E}(M)$.

Next, let us introduce "generalized velocity vectors" with components $\omega^{\Gamma}$ :

$$
\begin{aligned}
\boldsymbol{\omega}^{\Gamma} & =\frac{\Omega^{\Gamma}}{d z}=\Omega_{\alpha_{1} \ldots \alpha_{E}}^{\Gamma} \frac{\partial\left(x^{\alpha_{1}} x^{\alpha_{2}} \ldots x^{\alpha_{E}}\right)}{\partial\left(z^{1} z^{2} \ldots z^{E}\right)} \\
d z & =d z^{1} \wedge d z^{2} \wedge \ldots \wedge d z^{E} .
\end{aligned}
$$

In the above expression (9), $\frac{\partial\left(x^{\alpha} 1 x^{\alpha} 2 \ldots x^{\alpha} E\right)}{\partial\left(z^{1} z^{2} \ldots z^{E}\right)}$ stands for the Jacobian of the transformation from coordinates $\left\{x^{\alpha}\right\}$ over the manifold $M$ to coordinates $\left\{z^{a}\right\}$ over the embedded manifold $E$. The Jacobians provide a natural basis for the corresponding space. Note that $\Gamma$ in the above expression is a place holder for a particularly interesting vector in that space or a specific coordinate. For example, if these $\Omega^{\Gamma}$ forms are taken to be the Jacobians, then we will use the short hand notation $Y^{\Gamma}=\frac{\partial\left(x^{\alpha} 1 x^{\alpha} \ldots x^{\alpha} E\right)}{\partial\left(z^{1} z^{2} \ldots z^{E}\right)}$ with $\Gamma$ being the integer valued labeling function of the anti-symmetric ordered set, e.g., lexicographically, $\left\{\left\{\alpha_{1}, \alpha_{2} \ldots \alpha_{E}\right\}: \alpha_{i} \in\{1, \ldots, m\}\right\}$ corresponding to an element there; therefore, with values in the range $\{1, \ldots, D\}$. Thus, while $\Omega^{\Gamma}$ stands for a particularly interesting vector in that space $\Lambda^{E}(M)$, its coordinates, in that space, then are $\Omega_{\alpha_{1} \ldots \alpha_{E}}^{\Gamma}$. The general $\Omega^{\Gamma}$ becomes $Y^{\Gamma}$ when the coordinates are the corresponding Kronecker-delta functions: $\Omega_{\alpha_{1} \ldots \alpha_{E}}^{\Gamma}=\delta_{\Gamma, \alpha}$.

In the case (8) the pull-back of an $E$-form $\Omega^{\Gamma}$ must be proportional to the volume $d z=d z^{1} \wedge d z^{2} \wedge \cdots \wedge d z^{E}$ over the manifold $E$, just as in the corresponding discussion after Equation (5):

$$
\begin{aligned}
\phi^{*}\left(\mathbf{\Omega}^{\Gamma}\right) & =\omega^{\Gamma} d z^{1} \wedge d z^{2} \wedge \cdots \wedge d z^{E}= \\
& =\Omega_{\alpha_{1} \ldots \alpha_{E}}^{\Gamma} \frac{\partial\left(x^{\alpha_{1}} x^{\alpha_{2}} \ldots x^{\alpha_{E}}\right)}{\partial\left(z^{1} z^{2} \ldots z^{E}\right)} d z^{1} \wedge d z^{2} \wedge \cdots \wedge d z^{E} .
\end{aligned}
$$

Therefore, it is suitable for integration over the E-manifold. Thus, the action for the embedding $\phi$ is:

$$
S[\phi]=\int_{E} L(\vec{\phi}, \boldsymbol{\omega}) d z=\int_{E} \phi^{*}(\boldsymbol{\Omega})=\int_{E} A_{\Gamma}(\vec{\phi}) \boldsymbol{\omega}^{\Gamma} d z .
$$

This is a homogeneous function in $\omega$ and is reparametrization (diffeomorphism) invariant with respect to the diffeomorphisms of the E-manifold. If one relaxes the linearity 
$L(\vec{\phi}, \boldsymbol{\omega})=\phi^{*}(\boldsymbol{\Omega})=A_{\Gamma}(\vec{\phi}) \boldsymbol{\omega}^{\Gamma}$ in $\boldsymbol{\omega}$, then the canonical expression for the first-order homogeneous Lagrangian gives:

$$
\begin{aligned}
L(\vec{\phi}, \omega) & =\sum_{n=1}^{\infty} \sqrt[n]{S_{n}(\omega, \ldots, \omega)}= \\
& =A_{\Gamma} \omega^{\Gamma}+\sqrt{g_{\Gamma_{1} \Gamma_{2}} \omega^{\Gamma_{1}} \omega^{\Gamma_{2}}}+\ldots \sqrt[m]{S_{m}(\omega, \ldots, \omega)} .
\end{aligned}
$$

At this point, there is a strong analogy between the relativistic point particle and a general $p$-brane. However, there is a difference in the number of components. In particular, $\vec{x}, \vec{v}$, and $\vec{\phi}=\vec{x} \circ \phi$ have the same number of components $(m=\operatorname{dim}(M))$, however, the "generalized velocity" $\omega$ has a bigger number of components $D=\left(\begin{array}{c}m \\ E\end{array}\right) \geq m$ that are related to the Jacobians (9) [50]. The linearly independent general elements of this space are labeled with the index $\Gamma$ to allow index contraction with a relevant interaction field $A_{\Gamma}, g_{\Gamma_{1} \Gamma_{2}}$, or $S_{\Gamma_{1} \ldots \Gamma_{n}}^{n}$.

Some specific examples of $p$-brane theories correspond to the following familiar Lagrangians in theoretical and mathematical physics:

- The Lagrangian for a 0-brane (relativistic point particle in an electromagnetic field, $\operatorname{dim} E=1$ and $\left.\omega^{\Gamma} \rightarrow v^{\alpha}=\frac{d x^{\alpha}}{d \tau}\right)$ is:

$$
\begin{gathered}
L(\vec{\phi}, \boldsymbol{\omega})=A_{\Gamma} \omega^{\Gamma}+\sqrt{g_{\Gamma_{1} \Gamma_{2}} \omega^{\Gamma_{1}} \omega^{\Gamma_{2}}} \rightarrow L(\vec{x}, \vec{v}) \\
L(\vec{x}, \vec{v})=q A_{\alpha} v^{\alpha}+m \sqrt{g_{\alpha \beta} v^{\alpha} v^{\beta}} ;
\end{gathered}
$$

- The Lagrangian for a 1-brane (strings, $\operatorname{dim} E=2)[5]$ is:

$$
L\left(x^{\alpha}, \partial_{i} x^{\beta}\right)=\sqrt{Y^{\alpha \beta} Y_{\alpha \beta}},
$$

using the notation:

$$
\begin{aligned}
\omega^{\Gamma} \rightarrow Y^{\alpha \beta}=\frac{\partial\left(x^{\alpha}, x^{\beta}\right)}{\partial(\tau, \sigma)}= & \operatorname{det}\left(\begin{array}{cc}
\partial_{\tau} x^{\alpha} & \partial_{\sigma} x^{\alpha} \\
\partial_{\tau} x^{\beta} & \partial_{\sigma} x^{\beta}
\end{array}\right)= \\
& =\partial_{\tau} x^{\alpha} \partial_{\sigma} x^{\beta}-\partial_{\sigma} x^{\alpha} \partial_{\tau} x^{\beta} .
\end{aligned}
$$

In this case, the index $\Gamma$ for labeling the components of the generalized velocity vector $\omega^{\Gamma}$ corresponds to the set of pairs $\{\alpha, \beta\}$ out of $m$ elements. For example, for $m=4$ this will be $4 ! / 2 !^{2}=6$ not 4 like for the standard velocity vector in $M$;

- The Lagrangian for a general $p$-brane has the Dirac-Nambu-Goto term (DNG) [51]:

$$
L\left(x^{\alpha}, \partial_{E} x^{\beta}\right)=\sqrt{Y^{\Gamma} Y_{\Gamma}} .
$$

Notice that most of the Lagrangians above, except for the relativistic particle, are restricted only to gravity-like interactions. In the case of the charged relativistic particle, the electromagnetic interaction is very important. The corresponding interaction term for $p$-branes is known as a Wess-Zumino term [52].

The above discussion can be viewed as a justification of important class of model Lagrangian systems via the principle of reparametrization invariance when applied to the mechanics of point particle as well as to the mechanics of extended objects. The principle leads naturally to important, well-known, and studied electromagnetic-like $(n=1)$ and gravity-like $(n=2)$ interaction terms. However, the framework also suggests new possible fields $(n>2)$. Thus, it is important to investigate the additional higher-order terms and their relevance to our physical reality. 


\section{Classical Forces Beyond Electromagnetism and Gravity}

So far, the focus of the paper has been to justify and encourage the study of models based on first-order homogeneous Lagrangians by emphasizing their general properties, their potential to provide a mathematical justification of the observed macroscopic physical reality, and along the way, to set the stage for the study of diffeomorphism invariant mechanics of extended objects by following the close analogy with the relativistic point particle. Consequently, it is important to understand these new terms in the canonical expression of the first-order homogeneous Lagrangians (6). In this respect, this section discusses the implications of such interaction terms beyond electromagnetism and gravity as given by (6).

Before we go into more detail on this exploration, it should be pointed out that the results which follow are somewhat similar, in the sense that such behavior is unusual for a standard classical particle, to what has been observed in the a la Bogoslovsky studies. In particular, in the Bogoslovsky's model the mass dependent terms in the corresponding energy-momentum dispersion relation receive additional parameter $b$-dependence due to the presence of the specific conformal factor, in this respect one should probably talk, for example, about renormalization of the kinematical mass. Another effect observed is the presence of zero momentum even when the velocity is zero $[45,48]$. The work of Bogoslovsky will not be considered in any further details since the focus of the current study is on a different mathematical structure of the first-order homogeneous Lagrangians than the one studied by Bogoslovsky. In this respect the purpose of this section is to emphasize the unusual behavior when going beyond standard gravity and electromagnetism, that is pure $S_{n}$ type interactions with $n>2$ rather than confirming or comparing the details of such unusual effects.

Let us begin our journey by recognizing that one can circumvent the linear dependence, $\left(\operatorname{det}\left(\frac{\partial^{2} L}{\partial v^{\alpha} \partial v^{\beta}}\right)=0\right)$ due to the reparametrization symmetry, of the equations of motion derived from $L=\sqrt[n]{S_{n}(\vec{v}, \ldots, \vec{v})}$ by adding an extra set of equations $\left(\frac{d L}{d \tau}=0\right)$. This way the equations of motion derived from $L=\sqrt[n]{S_{n}(\vec{v}, \ldots, \vec{v})}$ and $\frac{d L}{d \tau}=0$ are equivalent to the equations of motion derived from $L=S_{n}(\vec{v}, \ldots, \vec{v})$. This is similar to the discussion at the end of Section 2. As noticed before, this is a specific choice of parametrization such that $g_{\alpha \beta}(x) v^{\alpha} v^{\beta}$ is constant.

If one focuses on a specific $n^{\text {th }}$-term of re-parametrization invariant Lagrangian (6), that is, $L=\left(S_{n}(v)\right)^{1 / n}$ in the parametrization gauge $S_{n}(v)=$ const then the equations of motion are:

$$
S_{n / \alpha / \beta} \frac{d v^{\beta}}{d \tau}=S_{n, \alpha}-S_{n / \alpha, \beta} v^{\beta} .
$$

Here $S_{n, \alpha}$ denotes partial derivative with respect to $x^{\alpha}$ when $S_{n / \alpha}$ indicates partial derivative with respect to $v^{\alpha}$. From this expression, it is clear that $n=2$ is a model that results in velocity independent symmetric tensor $S_{n / \alpha / \beta}(v)$ that can be associated with the metric tensor. Usually, such a metric tensor is assumed invertible and therefore the differential equations can be written in the form acceleration as a function of velocity and position. However, in general, the left-hand side $S_{n / \alpha / \beta}(v)$ goes as $v^{n-2}$ while the right-hand side as $v^{n}$ which will result in the general behavior that the acceleration grows as $v^{2}$ at most. This is consistent with the known velocity dependence of the equation of the geodesics as well as the equation of the geodesic deviations. The growth is not usually an issue since there is a limitation on the magnitude $v<c$ due to the finite propagation speed. Thus, for a suitably chosen units, $c=1$ one should have $\left|v^{\alpha}\right| \leq 1$. However, if $n>2$ and if the maximum speed limit 1 is reached along one coordinate, then there could be issues for keeping the system at rest with respect to another coordinate direction since a term like $1 / v^{n-2}$ will grow towards infinity when $v \rightarrow 0$. For homogeneous, isotropic, and static $S_{n}$ background field, with invertible symmetric tensor $S_{n / \alpha / \beta}(v)$, a particle described by (11) should be moving with a constant non-zero speed in all directions, in order to avoid infinite acceleration effects. 


\subsection{Simplest Pure $S_{n}(v)$ Lagrangian Systems}

To further illustrate our point above and to gain a better understanding of the $S_{n}(v)$ terms, let us consider the simplest possible pure $S_{n}(v)$ Lagrangian systems by assuming:

- Curvilinear coordinate system such that: $S_{n}(v)=f(t, r, w, u)$ where $w=d x^{0} / d \tau$ and $u=d r / d \tau$;

- $\quad$ Static fields, that is: $S_{n}(v)=f(r, w, u)$;

- Inertial coordinate system in the sense of Newtonian like space and time separation, that is: $S_{t \ldots t r . . r}=0$ except for $S_{t \ldots t}$ and $S_{r \ldots r}$ components.

Thus, the expression for $S_{n}(v)$ takes upon the following form:

$$
S_{n}(r, w, u)=\psi(r) w^{n}+\phi(r) u^{n} .
$$

Notice that here the symbols $u$ and $w$ are used to denote the spatial $r$ and time-like velocity coordinates instead of the previously used symbol $v$. Later in the discussion, this symbol $v$ will be used to denote the spatial speed in the Newtonian limit using coordinatetime parametrization $v=d r / d t$. This way the corresponding equations of motion for $L=S_{n}(r, w, u)$ are:

$$
\begin{aligned}
\frac{d u}{d \tau} & =-\frac{u^{2} \phi^{\prime}(r)}{(n-1) \phi(r)}+\frac{1}{u^{n-2}} \frac{w^{n} \psi^{\prime}(r)}{n(n-1) \phi(r)}, \\
\frac{d w}{d \tau} & =-\frac{w u \psi^{\prime}(r)}{(n-1) \psi(r)} .
\end{aligned}
$$

One can recognize the connection of the fields $\psi(r)$ and $\phi(r)$ to the energy and linear momentum of a particle by looking at the generalized momentum: $p_{\alpha}=\frac{\partial L}{\partial v^{\alpha}}$. In particular, $\psi(r)$ is related to the energy of the particle $E=p_{0}=\frac{\partial L}{\partial w}$, especially when considering $\tau=x^{0}=c t$ in co-moving frame $u / w=v / c \approx 0$ using coordinate-time parametrization where $w=1$. In this respect, if the energy of the particle is conserved then $\psi(r)=$ constant and therefore $\psi^{\prime}(r)=0$. The "radial" acceleration at macroscopic scales is then:

$$
a_{r}=\frac{d v}{d t}=-\frac{v^{2} \phi^{\prime}(r)}{(n-1) \phi(r)} .
$$

Here, the speed of light $c$, the maximum speed of propagation cancels out and $u=$ $d r / d(c t)=v / c$ is related to the spatial speed $v$. If $n=2$ and $\phi(r)=b r$ then one recovers the usual kinematical expression for the normal acceleration of a particle moving in a circular orbit $\left(a_{n}=v^{2} / r\right)$.

In general, however, depending on the specifics of the model and details of $\phi(r)$ one may obtain deviations from the flat space or the metric model for gravity. Such new models and forces could be relevant at large/cosmological scales where the dark-matter problem manifests itself in the deviation of the kinematical acceleration from the anticipated gravitational acceleration in galaxies and clusters of galaxies. Depending on the sign of $\phi^{\prime}(r) / \phi(r)$ this term could be "dissipative" in the sense that the system will settle at $v=0$ after a sufficiently long time if the sign of $\phi^{\prime}(r) / \phi(r)$ is positive. If the sign is negative then one has "repulsive gravity" that could be relevant to the dark-energy problem since the system will have an unstable $v=0$ configuration. Any further speculations about this equation are poorly justified without any underlining theory that predicts $\phi(r)$ and compares it to experimental observations.

At the microscopic scale, however, one may have $\psi^{\prime}(r) \neq 0$. This could suggest that the energy $p_{0}$ may not be conserved due to the interaction of the particle with the environment; thus, it may be subject to energy exchange. However, $p_{0}$ should nevertheless be conserved since the model under consideration has no explicit coordinate-time dependence. This can 
be illustrated using the equation for $w(14)$. The equation can be rewritten in a form that makes it easy to be integrated and to see the conservation of the energy $p_{0}$ :

$$
\begin{aligned}
d \ln (w) & =-\frac{1}{(n-1)} d \ln (\psi) \Rightarrow \\
\psi(r) w^{n-1} & =\text { constant }=p_{0} / n
\end{aligned}
$$

The corresponding generalized linear momentum then will be:

$$
p_{r}=n \phi(r) u^{n-1}=\frac{\phi(r)}{\psi(r)} p_{0}\left(\frac{v}{c}\right)^{n-1}
$$

This shows that the model considered does not have the Bogoslovsky non-zero rest momentum behavior and the effects due to $S_{n}$ fields on the velocity-momentum relation seems to be non-linear and highly "relativistic".

Looking back at the first Equation (13) for $u$, when $n=2$ the spatial force has two parts, one is velocity independent force proportional to $\propto \frac{p_{0}^{2} \psi^{\prime}(r)}{2^{3} \psi(r)^{2} \phi(r)}$, and the other part could be "dissipative" if the sign of $\phi^{\prime}(r) / \phi(r)$ is positive or "repulsive gravity" if the sign of $\phi^{\prime}(r) / \phi(r)$ is negative as discussed earlier.

For $n>2$ the physics interpretation of the equation of motion (13) leads to unusual behavior:

$$
\frac{(n-1)}{w^{2}} \frac{d u}{d \tau}=-\frac{v^{2} \phi^{\prime}(r)}{c^{2} \phi(r)}+\frac{c^{n-2}}{v^{n-2}} \frac{\psi^{\prime}(r)}{n \phi(r)} .
$$

It seems that an observer cannot detect a particle to be in complete rest $(u / w=$ $v / c=0)$ for a finite time interval $\Delta t$. If the speed of a particle was zero $(v=0)$ at some moment then the particle should have an infinite acceleration $d u / d \tau$ at that moment since $d u / d \tau \propto w^{2} v^{2-n} \rightarrow \infty$. Thus, the particle will instantaneously leave the state $v=0$ for a non-zero velocity state rather than staying in the zero velocity state. Depending on the details of the fields $\psi^{\prime}(r)$ and $\phi^{\prime}(r)$ there may not be a zero external force configuration for such a particle in general. Nevertheless, specific fields $\psi^{\prime}(r)$ and $\phi^{\prime}(r)$ may allow for zero acceleration state $d u / d \tau=0$ and non-zero spatial velocity state $u / w=v / c \neq 0$ :

$$
v^{n}=\frac{c^{n} \psi^{\prime}(r)}{n \phi^{\prime}(r)}
$$

However, such state would imply that the observer cannot be in the co-moving frame of the particle anymore since the coordinate time $t$ will not be synchronized with the "proper-time" $\tau$ of the particle $d w / d \tau \neq 0$ and thus $w=d x^{0} / d \tau \neq$ constant.

The above-discussed pathology is strikingly similar to the manner in which quantum mechanical particles behave: Particles cannot be localized with speed as close to zero as one wishes to; even more, the conservation of energy needs to be amended due to external fields (16). Therefore, such terms with $n>2$ may play important role in the understanding of the mechanism behind the inflation driven early stage of the universe, as well as in the derivation of the Dirac equation containing fundamental sub-atomic interactions beyond electromagnetism and gravity (for preliminary discussion see $[38,39,53]$ ). It should be noted that such pathological behavior may be attributed to radiation-reaction or the problem of self-force (see i.e., Abraham-Lorentz and Abraham-Lorentz-Dirac forces [54,55]) which is resolved in quantum electrodynamics via renormalization that requires adding higher order counter-terms to the Lagrangian [56].

Not being able to observe a particle at rest seems somewhat in contradiction to our classical physics reality. However, the more appropriate Lagrangian should take into account that "empty space" has Minkowski geometry:

$$
L=m \sqrt{\eta_{\alpha \beta} v^{\alpha} v^{\beta}}+\kappa \sqrt[n]{S_{n}(\vec{v}, \ldots, \vec{v})}
$$


Here $\eta_{\alpha \beta}=(1,-1, \ldots,-1)$ is the Lorentz invariant metric tensor. For Lagrangians that contain gravity $\left(S_{2}(v)\right.$ term) the problem for spatial velocity limit $v \rightarrow 0$ does not exist as discussed above for the case $n=2$. In the non-relativistic limit $(v / c \rightarrow 0)$, the present model of pure $S_{n}$ interaction in Minkowski spacetime results in an acceleration $\frac{d v}{d \tau}$ that is the same up to $O\left(v^{2}\right)$ terms for $L=$ const parametrization as well as for $\sqrt{\eta_{\alpha \beta} v^{\alpha} v^{\beta}}=$ const parametrization. Thus the non-relativistic limit cannot distinguish these two choices of parametrization.

\subsection{Choice of Proper Time Parametrization}

It was mentioned earlier that for parameter independent homogeneous Lagrangians of order $\alpha$, one has $h=(\alpha-1) L$ and thus $d L / d \lambda=0$ except for $\alpha=1$ that singles out first-order homogeneous Lagrangians. When working with re-parametrization invariant Lagrangian model, one can choose parametrization so that $L d \lambda=d \tau$ or effectively thinking of $L(x, v)=$ const. This brings the homogeneous Lagrangians of first order back in the family $d L / d \lambda=0$.

This appears to be the choice of parametrization to be made $\lambda \rightarrow \tau$ if the structure of $L$ is not known. However, it seems that $\sqrt{g_{\alpha \beta} v^{\alpha} v^{\beta}}=$ const is preferred [22] as physically more relevant due to its connection to the lifetime of unstable elementary particles. Especially, due to the lack of experimental evidence that the lifetime of charged elementary particles is affected by the presence of electromagnetic fields. This can be related to the observation that for any Lagrangian of the form $L=v^{\mu} A_{\mu}(x, v)$, where $x$ is space-time coordinate and $v$ is a world-line velocity vector (4-vector for $3+1$ space-time), one can define a velocity dependent symmetric tensor $g_{\alpha \beta}(x, v)=\frac{1}{2}\left(A_{\alpha / \beta}(x, v)+A_{\beta / \alpha}(x, v)\right)$ where $A_{\beta / \alpha}(x, v)$ denotes partial derivative with respect to $v^{\alpha}$ of $A_{\beta}(x, v)$. Then one can show that $\frac{d}{d \lambda}\left(v^{\alpha} g_{\alpha \beta}(x, v) v^{\beta}\right)=0$ along the trajectory determined by the Euler-Lagrange equation for $L=v^{\mu} A_{\mu}(x, v)$ - just like the usual geodesic equation of motion as in the discussion presented in Section 2. This symmetric tensor $g_{\alpha \beta}(x, v)$ does not depend on the velocity independent electromagnetic vector potential $A_{\mu}(x)$ and thus the length of the vector as calculated with $g_{\alpha \beta}(x, v)$ is not affected by the presence of electromagnetic interaction. Therefore, a proper time parametrization that coincides with the traditional definition: $d \tau=\sqrt{g_{\alpha \beta}(x, v) d x^{\alpha} d x^{\beta}}$ can be introduced.

The name of this special choice of $\tau$ parametrization derives from the fact that it is generally covariant and thus independent of the observer's coordinate system and can be interpreted as the passing of time measured in the rest frame of the system under study. Therefore, it is often of the form $d \tau=\sqrt{g_{00}(t)} d t$ and thus can be integrated along the laboratory coordinate time $t$. The laboratory coordinate time $t$ is up to the observer at rest as part of the laboratory measuring tools for various processes. Unfortunately, for first-order homogeneous Lagrangians, one has $v^{\alpha} g_{\alpha \beta}(x, v) v^{\beta}=0$ because $A_{\mu}(x, v)$ is a homogeneous function of zero degree and thus $v^{\beta} A_{\mu / \beta}(x, v)=0$. This seems to make it difficult to define the proper time parametrization in the usual way: $d \tau=\sqrt{g_{\alpha \beta}(x, v) d x^{\alpha} d x^{\beta}}$ for such first-order homogeneous Lagrangians $L=v^{\mu} A_{\mu}(x, v)$.

In this respect, for first-order homogeneous Lagrangians in the velocity, it is not clear if one has to choose "proper time" parametrization so that $L=$ const, or $\sqrt{g_{\alpha \beta^{\alpha} v^{\alpha} v^{\beta}}}=$ const, or $L-A_{\mu}(x) v^{\mu}=$ const. The choice $L-A_{\mu}(x) v^{\mu}=$ const may very well be a suitable choice since the weak and the strong forces do have an effect on the lifetime of elementary particles; for example, neutrons are unstable in free space but stable within nuclei. In connection to this, note that the other terms beyond gravity $\left(S_{n}\right.$ with $\left.n>2\right)$ are seemingly related to the internal degrees of freedom of the elementary particles. This should become more clear once a non-commutative quantization $(v \rightarrow \gamma)$ is applied to the re-parametrization invariant Lagrangian, which will be discussed elsewhere (for some preliminary results see $[38,53])$. Unfortunately, it is not clear how to extract the $A_{\mu}(x) v^{\mu}$ component of any first-order homogeneous Lagrangian $L$ mathematically, which is applicable to a physically 
relevant process, that is not assuming electromagnetic interaction a priory. Mathematically, one can extract $A_{\mu}(x)$ from first-order homogeneous Lagrangian $L$ by considering $A_{\mu}(x)=$ $L(x, v)_{/ \mu}=p_{\mu}$ at $v^{\alpha} \rightarrow 0$; however, physically $v^{0}$ should never be zero. Nevertheless, in the case of the Simplest Pure $S_{n}(v)$ Lagrangian Systems discussed in the previous subsection, one can define "proper time" parametrization under certain conditions.

Based on the general discussion after Equation (11) and on the specific example Equation (13), the condition for reasonable parametrization such as "proper time" for $S_{n}(v)$ is surprisingly restrictive. It demands $n=2$ so that the laboratory clock could be at rest with respect to the particle studied. If $n>2$ there is this pathological behavior that moves the particle "instantaneously" away from the rest frame of the clock. Thus, only $n=2$ allows for a rest frame within the model Lagrangians discussed. Then by using the conservation of $p_{0}(16)$ one has: $2 \psi(r) c d t=p_{0} d \tau$ where almost everything is a constant $(c$ and $\left.p_{0}\right)$ and $\psi(r)$ seems to be related to the gravitational potential at the location $r$ where the particle is. In conclusion, it seems that "proper time" parametrization is only possible for $n=2$ systems based on the analyses of the Simplest Pure $S_{n}(v)$ Lagrangian Systems and the discussion above. Thus, gravity is essential for the notion of the "proper time" parametrization and no other Simple $S_{n}(v)$ Lagrangian System provides an alternative parametrization that makes sense as the passing of time in the rest frame of a particle.

To conclude this section, one may naively extrapolate the scale at which such new forces may be dominant. Considering that electromagnetic forces are relevant at an atomic and molecular scale when gravity is dominating the solar system and at galactic and cosmological scales, then one may deduce that terms beyond gravity may be relevant at galactic and intergalactic scales. Along this line of reasoning, a possible determination of the structure of such forces from the velocity distribution of stars in galaxies is an interesting possibility. In this respect, such forces can be of relevance to the dark matter and dark energy cosmology problems. The pathological $d v / d \tau \rightarrow \infty$ when $v \rightarrow 0$, behavior of pure $S_{n}$ for $n>2$ interactions could also be of relevance to inflation models. Finally, as already mentioned, such terms are essential for bringing in fields beyond the electromagnetic fields into the Dirac equation when considering the quantization of the first-order homogeneous Lagrangians in the velocity.

\subsection{Fictitious Accelerations in Un-Proper Time Parametrization}

In the previous section, we discussed the concept of proper time parametrization. The coordinate time is evidently another choice of time parametrization. In general, if the action is reparametrization invariant then one should be able to use any choice of time parametrization for a process. However, if the action is not reparametrization invariant then one may find a puzzling phenomenon due to the choice of time parametrization for a process. The presence of a fictitious acceleration in un-proper time parametrization of non-reparametrization invariant action is the topic of the current section.

In the framework of Special and General Relativity, Carlip derived that retardation effects resulting from velocity dependent field potentials give rise to forces that are linear (for electromagnetism) and quadratic (for gravity) extrapolations pointing towards the instantaneous source location [49]. Note that this remarkable result is about the force on a test particle that does not alter (by definition) the overall field produced by the source.

The superposition of fields is a hallmark of the Maxwell equations for electromagnetism, however, this is not the case for gravity. Therefore, the overall gravitational field of a large realistic gravitationally bound system is somewhat more complicated. However, for a realistic two body system, the superposition of two or more fields does not represent the correct gravitational field needed to assess the motion of the bodies. Within the classical Lagrangian approach, the simplest option that may show some superposition-like properties could be based on the familiar quadratic Lagrangian $L_{2}(x, v)=g_{\mu v} v^{\mu} v^{v}$. For a point 
particle at point $x_{0}^{\mu}$ in the space-time such Lagrangian can clearly exhibit superposition up to a leading order term due to $N$ sources that are far from each other so that:

$$
g^{\mu v}=\eta^{\mu v}+\sum_{i=1}^{N} g_{i}^{\mu \nu}\left(x_{0} ; x_{i}, v_{i}\right), \quad g_{i}^{\mu v}\left(x_{0} ; x_{i}, v_{i}\right)=\frac{2 G m_{i}}{\left(r\left(v_{i}\right)\right)^{3}} \sigma_{i}^{\mu} \sigma_{i}^{v} .
$$

In the above equation we follow Carlip's notation where $r\left(v_{i}\right)$ is the velocity dependent distance that is covariant. The subscripts $i>0$ is reserved for the point sources, while $i=0$ or no subscript is reserved for the test particle location. Note that no superscripts for $x_{i}$ and $v_{i}$ indicate the position and velocity four-vectors in $4 \mathrm{D}$ space-time. Finally, due to the linearity of the Euler-Lagrange equations of motion derived from the action $A_{2}=\int L_{2}(x, v) d t$ with respect to the corresponding Lagrangian it follows that there will be a superposition of the gravitational fields. Notice that the coordinate time of the test particle $x_{0}^{0}=c t$ is naturally the time parameter to describe the evolution within the action integral. The above setup has a potential to agree with Carlip's derivations in [49].

Now, let us consider the case when there are two bodies with a significant deviation from the test-particle idea. In this case, one may want to start with the construction above for the metric field, since it may stand the chance of almost linear superposition when the bodies are sufficiently far apart. However, in the setting up of the action $A=\int L(x, v) d \tau$ one has to decide on what would be the meaning of the time-like parameter $\tau$. For example, in the case of the Planet-Moon system, should the time be the Planet time or the Moon time or the center of mass time? And if we choose the center of mass, would the center of mass point-like potential be a good enough approximation of the true gravitational field? In a true general relativistic approach the choice of coordinate system is irrelevant but this path is long and difficult to walk. Instead, let us consider an equivalent Lagrangian formulation that has reparametrization invariant action: $A_{1}=\int L_{1}(x, v) d \tau=\int \sqrt{L_{2}(x, v)} d \tau$. Note that now the linear superposition of gravitational fields is most likely violated. Since this is parametrization independent, we can consider $\tau$ to be any coordinate time we desire. Such expression for the gravitational part of the Lagrangian is a standard choice in Special and General Relativity along with the condition $g_{\mu \nu} v^{\mu} v^{\nu}= \pm 1$, where the choice \pm depends on the choice of the metric signature. This choice means that the proper-time $\tau$ of the test particle has been chosen as the overall time parameter. As a result one has $\dot{L}=0$ along the path of the test particle and the equations of motion derived from $A_{2}$ or $A_{1}$ are the same. What happens if one relaxes this choice $(\dot{L}=0)$ ?

If one derives the Euler-Lagrange equations, one would find that the equations derived from the action: $A_{f}=\int f(L(x, v)) d t$ are:

$$
\frac{d p_{\mu}}{d t}=\partial_{\mu} L-\frac{f^{\prime \prime}}{f^{\prime}} \dot{L} p_{\mu}, p_{\mu}=\frac{\partial L}{\partial v^{\mu}} \Rightarrow \frac{d p_{\mu}}{d t}=\partial_{\mu} L+\frac{\dot{L}}{2 L} p_{\mu} \text {, when } f(L)=\sqrt{L} \text {. }
$$

From the above expressions one can see that if the time parameter is such that $\dot{L}=0$ then the extra term in the right-hand-side will vanish for any reasonable action $A_{f}$. Thus, all such Euler-Lagrange equations will be equivalent. While this is highly desirable, one is very likely to use unsuitable time parameter and/or metric field due to deviation of nature from our ideal abstract model and their inability to account for everything. Thus, $\dot{L} \neq 0$ is a very likely situation resulting in a fictitious force.

For deviations from Einstein GR based on the Integrable Weyl geometry, in particular the Scale Invariant Vacuum (SIV) theory [24], one often considers a metric $g^{\mu v}=\lambda^{-2} g_{\mathrm{GR}}^{\mu v}$ along with a metric connection $\kappa_{\mu}=-\partial_{\mu} \ln \lambda$. In SIV, one has $\lambda(t) \propto t^{-1}$ (only time dependence) and in the weak field limit of homogenous and isotropic space one has usually an extra acceleration: $\kappa_{0} \vec{v}=\vec{v} / t$, where $t$ is the cosmic time since the Big Bang [28]. If we adopt the same view about our model metric $g^{\mu v}$ in $L$, then we have:

$$
\frac{d v_{\mu}}{d t}=\partial_{\mu} L+\kappa_{0} v_{\mu} \text {, when } L=g_{\mu \nu} v^{\mu} v^{v}
$$


Thus, one can see the appearance of fictitious force that enhances the motion of a particle that acts as a non-conservative force. Such fictitious force seems to be reflecting the deviation of our models from the true reality. There could be a lot of things coming into interaction with our gravitating system as well as internal changes such as tidal effects that transfer rotational energy into internal energy and so on. In this respect the force is dissipative-like, since mechanical energy is transferred into internal energy of the system. This should not affect the overall gravitational field but our inability of utilizing the true proper time parametrization for the description of the system will bring in an apparent violation of the usual conservation laws. Thus, the use of un-proper (un-proper seems better name than improper, since any time parametrization should be ok, but only in proper time parametrization one will see clearly the conservation of familiar quantities) time parametrization results in extra fictitious terms.

To understand better the effect consider the original generalized momentum case (22) and $L=\lambda^{-2} L_{\mathrm{GR}}$ with $d\left(L_{\mathrm{GR}}\right) / d t=0$, which results in:

$$
\frac{d p_{\mu}}{d t}=\partial_{\mu} L+\kappa_{0} p_{\mu}, \quad \text { where } \kappa_{0}=-\dot{\lambda} / \lambda
$$

If we utilize the usual expectation for our model Lagrangian $L$ that leads to the usual energy and momentum conservation $\left(\partial_{t} L=0\right.$ and $\left.\partial_{\phi} L=0\right)$, that is, absence of explicit time and angle $\phi$ dependence for our model Lagrangian $L$. Then the corresponding energy, given by the quadratic Hamiltonian $\left(h \propto p_{\mu} v^{\mu}-L=g_{\mu \nu} v^{\mu} v^{v}\right)$ and angular momentum $(J)$ conservation equations are modified and result in the following new expressions:

$$
\begin{array}{r}
\frac{d h}{d t}=\frac{d\left(\lambda^{-2} L_{\mathrm{GR}}\right)}{d t}=2 \kappa_{0} h \Rightarrow \frac{\dot{h}}{h}=2 \kappa_{0}, \\
\frac{d J}{d t}=\partial_{\phi} L+\kappa_{0} J=\kappa_{0} J \Rightarrow \frac{\dot{J}}{J}=\kappa_{0} .
\end{array}
$$

The practical meaning of the new results is the possibility to observe non-conservation effects $\dot{h} \neq 0$ and $\dot{j} \neq 0$ when our experiment reaches accuracy resulting in fractional uncertainty compatible to $\kappa_{0} \delta t$ at high time resolution. Even though the expressions above are written as derivatives these are actually very small effects that accumulate over an extended period of observational data. These non-conservation effects are usually buried in much bigger fluctuations of the corresponding quantities as seen in actual astronomical observations that are usually explained by tidal effects and similar dissipative processes. Recent research results suggest possible new viewpoint for understanding and probably explaining puzzling measurement results within the Solar System-the paper is in preparation by the authors and in collaboration with Prof. M. Krizek. The main point here is the presence of such effects due to un-proper time parametrization of a process along with non-reparametriosation invariant action for that process.

\section{The Background Fields and Their Lagrangians}

The uniqueness of the interaction fields and their source types has been essential for the selection of the matter Lagrangian (10). The first two terms in the Lagrangian are easily identified as electromagnetic and gravitational interaction. The other terms describe new classical forces. It is not yet clear if these new terms are actually present in nature or not, so one shall not engage them actively in the following discussion but our aim is to start preparing the stage for such research and discussions. At this point, one has a theory with background fields since the equations for the interaction fields are not known. To complete the theory, one needs to introduce actions for these interaction fields.

One way to write the action integrals for the interaction fields $S_{n}$ in (10) follows the case of the $p$-brane discussion. There, one has been solving for $\phi: E \rightarrow M$ by selecting a Lagrangian that is more than a pull-back of an $E$-form over the manifold $M$. In a similar way, one may view $S_{n}$ as an $M$-brane field theory, where $S_{n}: M \rightarrow S_{n} M$ and $S_{n} M$ is the 
fiber of symmetric tensors of rank $n$ over $M$. This approach, however, cannot terminate itself since new interaction fields would be generated as in the case of $\phi: E \rightarrow M$.

Another way assumes that $A_{\Gamma}$ is an $n$-form. Thus, one may use the structure of the external algebra $\Lambda\left(T^{*} M\right)$ over $M$ to construct objects proportional to the volume form over $M$. For any $n$-form $(A)$ objects proportional to the volume form $\Omega_{\mathrm{Vol}}$ can be constructed by using operations in $\Lambda\left(T^{*} M\right)$, such as the external derivative $d$, external multiplication $\wedge$, and the Hodge dual $*$. For example, $A \wedge * A$ and $d A \wedge * d A$ are forms proportional to the volume form $\Omega_{\mathrm{Vol}}$.

The next important ingredient comes from the symmetry in the matter equation. That is, if there is a transformation $A \rightarrow A^{\prime}$ that leaves the matter equations unchanged, then there is no way to distinguish $A$ and $A^{\prime}$ by experiments and measurements via the matter that is obeying these equations. Thus the action for the field $A$ should obey the same symmetry (gauge symmetry) as those found in the equations of motion for the matter.

\subsection{Justifying the Electromagnetic Action}

Let us consider now the matter equation for $4 \mathrm{D}$ electromagnetic interaction which is $d \vec{v} / d \tau=F \cdot \vec{v}$ where $F$ is the 2-form obtained by differentiation of the 1-form $A(F=d A)$, and the gauge symmetry for $A$ is $A \rightarrow A^{\prime}=A+d f$ since the external differential operator $d$ obeys $d^{2}=0$. The reasonable terms, which can result in the volume form $\Omega_{\mathrm{Vol}}$ for the field Lagrangian $\mathcal{L}(A)$ of a 1-form field $A$, are then: $A \wedge * A, d A \wedge d A$, and $d A \wedge * d A$ and of course $A \wedge A \wedge A \wedge A$. The first and last terms do not conform with the gauge symmetry $A \rightarrow A^{\prime}=A+d f$ and the second term $(d A \wedge d A)$ is a boundary term since $d A \wedge d A=d(A \wedge d A)$ that gives $\int_{M} d(A \wedge d A)=A \wedge d A$ at the boundary of $M$; this term is interesting in the quantum Hall effect. Therefore, one is left with a unique action for fields based on a one-form $A=A_{\mu}(x) d x^{\mu}$ that respects the gauge symmetry of the corresponding Euler-Lagrange equations of motion for matter: $A \rightarrow A^{\prime}=A+d f$-this is exactly the electromagnetic field generated by moving charges $j^{\mu}=\rho v^{\mu}$ and described by the standard action:

$$
S[A]=\int_{M} d A \wedge * d A+A_{\mu} j^{\mu}=\int_{M} F \wedge * F+A_{\mu} j^{\mu} .
$$

Note that if $F$ was considered as a fundamental field rather than $A$ then in $4 \mathrm{D}$ one can also consider the term $F \wedge F$. However, as soon as one recognizes that $F=d A$ then this becomes the boundary term $(d A \wedge d A)$ discussed above. Furthermore, once $F=d A$ is recognized as a two-form and expressed in the coordinate basis $F_{\mu \nu} d x^{\mu} \wedge d x^{\nu}$ then one can also consider a gauge invariant term of the form: $F_{\mu v} d x^{\mu} \wedge d x^{v} \wedge *\left(d x^{\mu} \wedge d x^{v}\right)$ as part of the action. However, such a term is zero due to permutation symmetry since $W^{\nu \mu}=W^{\mu \nu}=d x^{\mu} \wedge d x^{\nu} \wedge *\left(d x^{\mu} \wedge d x^{v}\right) \propto \eta^{\mu \mu} \eta^{\nu v} d x^{0} \wedge d x^{1} \wedge d x^{2} \wedge d x^{3}$; thus, the antisymmetric $F$ and the symmetric $W$ contract to zero $\left(F_{\mu \nu} W^{\mu \nu}=0\right)$.

\subsection{Justifying the Einstein-Hilbert-Cartan Action}

For our next example, let us look at the terms related to the matter equations that involve gravity. There are two possible choices of matter equation. The first one is the geodesic equation $d \vec{v} / d \tau=\vec{v} \cdot \Gamma \cdot \vec{v}$ where $\Gamma$ is considered as a connection 1-form that transforms in the usual way $\Gamma \rightarrow \Gamma+\partial g$ under coordinate transformations by the group element $g$. This type of transformation, however, is not a "good" symmetry since restricting the gauge transformation $\Gamma \rightarrow \Gamma+\Sigma$ to transformations $\Sigma=\partial g$ such that $\vec{v} \cdot \Sigma \cdot \vec{v}=0$, would mean to select a subset of coordinate systems, inertial systems, for which the action $S$ is well defined and satisfies $S[\Gamma]=S[\Gamma+\Sigma]$. Selecting a specific class of coordinate systems for the description of a physical phenomenon is not desirable, so this option shall not be explored any further.

In general, the Euler-Lagrange equations assume a background observer who defines the coordinate system. For electromagnetism, this is acceptable since neutral particles are such privileged observers. In gravity, however, there is no such observer, and the equation 
for matter should be relational. Such an equation then is the equation of the geodesic deviation: $d^{2} \vec{\xi} / d \tau^{2}=\boldsymbol{R}(v, v) \cdot \vec{\xi}$, where $\boldsymbol{R}$ is a Lie algebra $(T M)$ valued curvature 2-form $\boldsymbol{R}=d \Gamma+[\Gamma, \Gamma]$. A general curvature 2 -form is denoted by $F \rightarrow\left(F_{\alpha \beta}\right)_{j}^{i}$. Here, $\alpha$ and $\beta$ are related to the tangential space $(T M)$ of the base manifold $M$. The $i$ and $j$ are related to the fiber structure of the bundle over $M$ where the connection $\left(\Gamma_{\alpha}\right)_{j}^{i}$ that defines $\left(F_{\alpha \beta}\right)_{j}^{i}$ is given. Clearly, the Riemann curvature tensor $R$ is a very special curvature because all of its indices are of the TM type. For that reason, it is possible to contract the fiber degree of freedom with the base manifold degree of freedom (indices). Thus, an action linear in $\boldsymbol{R}$ is possible. In general, one needs to consider a quadratic action $\left(F_{\alpha \beta j}^{i} \wedge * F_{\alpha \beta i}^{j}\right)$, i.e., trace of $F \wedge * F$.

Using the symmetries of the Riemann curvature tensor $\boldsymbol{R}\left(R_{\alpha \beta, \gamma \rho}=-R_{\beta \alpha, \gamma \rho}=\right.$ $\left.-R_{\alpha \beta, \rho \gamma}=R_{\gamma \rho, \alpha \beta}\right)$, one has two possible expressions that can be proportional to the volume form $\Omega$. The first expression is possible in all dimensions and can be denoted by $\boldsymbol{R}^{*}$, which means that a Hodge dual operation has been applied to the second pair of indices $\left(R_{\alpha \beta, *(\gamma \rho)}\right)$. The $\boldsymbol{R}^{*}$ action seems to be related to the Cartan-Einstein action for gravitation $S[R]=\int R_{\alpha \beta} \wedge *\left(d x^{\alpha} \wedge d x^{\beta}\right)$ [57]. The other expression is only possible in a four-dimensional space-time and involves full anti-symmetrization of $R\left(R_{\alpha[\beta, \gamma] \rho)}\right)$ denoted by $R^{\wedge}$. However, the fully anti-symmetric tensor $\boldsymbol{R}^{\wedge}$ is identically zero due to symmetry considerations related to the permutation group [58]. Since the symmetries of the equation of the geodesic deviation are encoded in the Riemann curvature tensor $\boldsymbol{R}$, then once again one arrives at the unique Einstein-Hilbert-Cartan action for gravity based on $\boldsymbol{R}$.

\section{Conclusions and Discussion}

In conclusion, the discussion in this paper showed the potency of the principle of reparametrization invariance when realized via the canonical-form of the first-order homogeneous Lagrangians in the velocity or generalized velocity by using the principle of one-to-one correspondence between an interaction field and its source to justify the fundamental interaction fields for the classical long-range forces via the geometrical concepts of embedding of manifolds as well as the natural differential structures over manifolds. In summary, the structure of the matter Lagrangian $(L)$ for extended objects, and in particular the point particle, have been discussed. Imposing reparametrization invariance of the Lagrangian based action $S=\int_{E} L(x, \omega)$ naturally leads to a first-order homogeneous Lagrangian. In its canonical form, the Lagrangian $L$ contains electromagnetic and gravitational interactions, as well as interactions that are not yet experimentally discovered but may be detected as a result of various efforts to address current discordances present between the different cosmological probes [26].

The fields $A_{\mu}(x)$ and $g_{\mu \nu}(x)$ associated with $n=1$ and $n=2$ homogeneous Lagrangians built from monomials in the velocities $S_{n}(v, \ldots, v)$ are clearly related to electromagnetic and gravitational interactions. Especially, if one recognizes that the gauge symmetry of these interaction fields are encoded in the 2-forms $F$ and $\boldsymbol{R}$ that naturally appear in the corresponding equations of motion-the Euler-Lagrange equation that corresponds to the Lorentz force $d \vec{v} / d \tau=q F \vec{v}$ for charged particles and the equation of the geodesic deviations for massive particles $d^{2} \vec{\xi} / d \tau^{2}=R(v, v) \vec{\xi}$.

If one extrapolates from the strengths of the two known classical long-range interactions, then it is natural to expect that the new terms in $L$ should be important, if present in nature at all, at big cosmological scales, such as those relevant to the dynamics of galactic and galactic clusters. Thus, perhaps relevant to the dark matter and dark energy phenomena [26]. Furthermore, the pathological behavior (18) discussed for the simplest model of $S_{n}(v, \ldots v)$ fields when $n>2$, may be relevant to the inflation processes in the early universe [26]. At microscopic scales such $n>2$, fields may be useful in justifying the interactions in the standard model of elementary particles upon suitable quantization that recovers the Dirac equation but with additional interactions beyond electromagnetism and gravitation. 
If one is going to study the new interaction fields $S_{n}(v, \ldots v), n>2$, then the guiding principles for writing field Lagrangians, as discussed in the examples of electromagnetism and gravity (Section 5), may be a useful starting point. Furthermore, it may be useful to apply the outlined constructions to gravity by considering it as a 3-brane in a 10dimensional target space $\left(g_{\alpha \beta}: M \rightarrow S_{2} M\right)$ and to compare it with the $10 D$ supergravity.

If such $S_{n}(v, \ldots v)$ related forces are not present in nature then one needs to understand why nature is not taking advantage of such possibilities. The choice of the canonical Lagrangian is based on the assumption of one-to-one correspondence between interaction fields and the type of sources. If one can show that any first-order homogeneous function can be written in the canonical form proposed, then this would be a significant step towards our understanding of the fundamental interactions in nature, especially if one can show that only $n=1$ and $n=2$ effective terms are needed. Note that an equivalent expression can be considered as well: $L=A_{\alpha}(\vec{x}, \vec{v}) v^{\alpha}$. This expression is simpler and is concerned with the structure of the homogeneous functions of order zero $A_{\alpha}(\vec{x}, \vec{v})$. In any case, understanding the structure of the homogeneous functions of any order seems to be an important mathematical problem with significant implications for physics.

\section{Examples and Exercises}

1. Show that $g_{\mu v} v^{\mu} v^{v}=$ constant along the trajectory of a particle is a necessary and sufficient condition for Euler-Lagrange equations corresponding to $S_{1}(1)$ and $S_{2}$ (2) to be equivalent to each other and to the geodesic equation (3). In the traditional case of velocity independent metric see [22];

2. Show that for any Lagrangian $L(x, v)$ that is a homogeneous function in the velocity $\vec{v}$ of order $n \neq 1$ the corresponding Hamiltonian function $h=v^{\alpha}\left(\frac{\partial L}{\partial v^{\alpha}}\right)-L$ is proportional to the Lagrangian, that is, $h=(n-1) L$;

3. Show that any time independent Lagrangian $L(\vec{x}, \vec{v})$, which is a homogeneous function in velocity $\vec{v}$ of order $n \neq 1$, is an integral of the motion with respect to the corresponding Euler-Lagrange equations for $L$;

4. Consider a Lagrangian that is a constant of the motion; that is, $d L / d \tau=0$. Show that any solution of the Euler-Lagrange equations for $L$ is also a solution for $\tilde{L}=f(L)$ under certain minor and reasonable requirements on $f$, such as $\tilde{L}=f(L) \neq 0$ and $\tilde{L}^{\prime}=f^{\prime} \neq 0$;

5. Show that if $v^{0}=d t / d \tau$ is well behaved $\left(v^{0} \neq 0\right.$ over the duration of the process studied) then the Euler-Lagrange equations for the reparametrization-invariant Lagrangian $L\left(x^{\mu}, v^{\mu}\right)=L\left(x^{\mu}, v^{i} / v^{0}\right) v^{0}$, where $i=1, \ldots, n, \mu=0,1, \ldots, n$ and $x^{0}=t, v^{i}=d x^{i} / d \tau, v^{0}=d t / d \tau$, are equivalent to the Euler-Lagrange equations for coordinate-time parametrization $(\tau=t)$ choice for $L\left(t, x^{i}, d x^{i} / d t\right)$. Hint: Use that $L\left(x^{\mu}, v^{i} / v^{0}\right)$ is a zero-order homogeneous function with respect to $v^{\mu}$ and notice the relationship between the Hamiltonian function $h$ for the initial Lagrangian $L\left(t, x^{i}, d x^{i} / d t\right)$ and the generalized momentum $p_{0}=\partial L / \partial v^{0}$ for the reparametrizationinvariant Lagrangian $L\left(x^{\mu}, v^{\mu}\right)=L\left(x^{\mu}, v^{i} / v^{0}\right) v^{0}$;

6. Show that $\sum_{\beta} v^{\beta} \frac{\partial^{2} L}{\partial v^{\alpha} \partial v^{\beta}}=0$ if $L$ is first-order homogeneous Lagrangian. Thus, $\operatorname{det}\left(\frac{\partial^{2} L}{\partial v^{\alpha} \partial v^{\beta}}\right)=0$, since in an extended space-time one usually expects $v^{0} \neq 0^{\prime}$

7. Consider the constraint $\sqrt{g_{\alpha \beta} v^{\alpha} v^{\beta}}=1$ implemented via a Lagrangian multiplier $\chi$ in the Lagrangian $L=q A_{\alpha} v^{\alpha}+(m+\chi) g_{\alpha \beta} v^{\alpha} v^{\beta}-\chi$. Show that the value of $\chi$ is required to be $\chi=-m / 2$ if $L=q A_{\alpha} v^{\alpha}+m \sqrt{g_{\alpha \beta}(x) v^{\alpha} v^{\beta}}$ and $L=q A_{\alpha} v^{\alpha}+(m+$ $\chi) g_{\alpha \beta} v^{\alpha} v^{\beta}-\chi$ are to result in the same Euler-Lagrange equations;

8. Show that the function $S_{n}(r, w, u)$ defined in Equation (12) is an integral of motion for the equations given by (13) and (14);

9. Consider the Lagrangian $L=m \sqrt{\eta_{\alpha \beta} v^{\alpha} v^{\beta}}+\kappa \sqrt[n]{S_{n}(\vec{v}, \ldots, \vec{v})}$, where $\eta_{\alpha \beta}=(1,-1, \ldots,-1)$ is the Lorentz invariant metric tensor. Show that in the non-relativistic limit $(v \rightarrow 0)$, the Euler- 
Lagrange equations for the acceleration $\frac{d v}{d \tau}$ are the same up to $O\left(v^{2}\right)$ terms whether $L=$ const or $\sqrt{\eta_{\alpha \beta} v^{\alpha} v^{\beta}}=$ const parametrization is imposed. Thus the non-relativistic limit cannot distinguish these two choices of trajectory parametrization;

10. Show that solutions of the Euler-Lagrange equations for $L=v^{\mu} A_{\mu}(x, v)$, where $x$ is a space-time coordinate and $v^{\mu}$ is a world-line velocity vector (4-vector for $3+1$ space-time), satisfy $\frac{d}{d \lambda}\left(v^{\alpha} g_{\alpha \beta}(x, v) v^{\beta}\right)=0$ for the velocity dependent metric $g_{\alpha \beta}(x, v)=\frac{1}{2}\left(A_{\alpha / \beta}(x, v)+A_{\beta / \alpha}(x, v)\right)$ with $A_{\beta / \alpha}(x, v)$ being a partial derivative with respect to $v^{\alpha}$ of $A_{\beta}(x, v)$;

11. Choose a specific Lagrangian $\tilde{L}(x, v)$ that is a homogeneous function of first order in $v$, then consider the Lagrangian $L=v^{\mu} A_{\mu}(x)+\sqrt{g_{\alpha \beta} v^{\alpha} v^{\beta}}$ where the fields $g_{\alpha \beta}(x)$ and $A_{\mu}(x)$ are defined via the following expressions: $A_{\mu}(x)=\frac{1}{2}(\tilde{L}(x, v)-$ $\tilde{L}(x,-v)) /\left.\mu\right|_{v=(1, \overrightarrow{0})}$ and $g_{\alpha \beta}(x)=\left.\frac{1}{4}(\tilde{L}(x, v)+\tilde{L}(x,-v))_{/ \alpha / \beta}^{2}\right|_{v=(1, \overrightarrow{0})}$. Compare the corresponding Euler-Lagrange equations of motions for $\tilde{L}$ and $L$. At what order $k$ of $O\left(v^{k}\right)$ are the differences?

Author Contributions: Conceptualization, V.G.G.; validation, V.G.G. and A.M.; formal analysis, V.G.G. and A.M.; writing—original draft preparation, V.G.G.; writing—review and editing, V.G.G. and A.M.; All authors have read and agreed to the published version of the manuscript.

Funding: This research received no external funding.

Institutional Review Board Statement: Not applicable.

Informed Consent Statement: Not applicable.

Data Availability Statement: Not applicable.

Acknowledgments: A.M. expresses his gratitude to his wife for her patience and support. V.G.G. is extremely grateful to his wife and daughters for their understanding and family support during the various stages of the research presented. Past preliminary research was performed at Louisiana State University and Lawrence Livermore National Laboratory. V.G.G. acknowledges past discussions on the topic with Carlos Castro Perelman and Haymaker, R., Rau, A.R.P., Kirk, P., Pullin, J., O'Connell, R., Torre, C., Baez, J., Nikolov, P.A., Prodanov, E.M., Dunne, G., Gould, L.I. and Singleton, D.

Conflicts of Interest: The authors declare no conflict of interest.

\section{References}

1. Kilmister, C.W. Lagrangian Dynamics: An Introduction for Students; Plenum Press: New York, NY, USA, 1967.

2. Goldstein, H. Classical Mechanics; Addison-Wesley: Boston, MA, USA, 1980.

3. Deriglazov, A.A. Classical Mechanics: Hamiltonian and Lagrangian Formalism, 2nd ed.; Springer: Cham, Switzerland, 2017.

4. Gràcia, X.; Pons, J.M. Singular Lagrangians: Some Geometric Structures Along the Legendre Map. J. Phys. A $2001,34,3047$. [CrossRef]

5. Klimenko, S.; Nikitin, I. Non-Critical String Theory: Classical and Quantum Aspects; Nova Science: New York, NY, USA, 2007.

6. Cariñena, J.F.; Ibort, L.A.; Marmo, G.; Sternd, A. The Feynman problem and the inverse problem for Poisson dynamics. Phys. Rep. 1995, 263, 153. [CrossRef]

7. Rund, H. The Hamilton-Jacobi Theory in the Calculus of Variations: Its Role in Mathematics and Physics; Van Nostrand: Huntington, NY, USA, 1966.

8. Lanczos, C. The Variational Principles of Mechanics, 4th ed.; University of Toronto Press: Toronto, ON, Canada, 1970.

9. Pauli, W. Theory of Relativity; Pergamon Press: New York, NY, USA, 1958.

10. Weyl, H.; Ehlers, J. Space, Time, Matter: Lectures on General Relativity; Springer: Berlin, Germany, 1993; 348p. (In German)

11. Borstnik, N.M.; Nielsen, H.B. Why odd-space and odd-time dimensions in even-dimensional spaces? Phys. Lett. 2000, B486, 314. [CrossRef]

12. van Dam, H.; Ng, Y.J. Why $3+1$ metric rather than $4+0$ or 2+2? Phys. Lett. 2001, B520, 159. [CrossRef]

13. Sachoglu, C. Fake R ${ }^{4}$ s, Einstein spaces and Seiberg-Witten monopole equations. Class. Quantum Grav. 2001, 18, 3287. [CrossRef]

14. Feynman, R.P.; Leightoon, R.B.; Sands, M. The Feynman Lectures on Physics; Addison-Wesley: Boston, MA, USA, 1965.

15. Gerjuoy, E.; Rau, A.R.P.; Spruch, L. A unified formulation of the construction of variational principles. Rev. Mod. Phys. 1983, 55, 725. [CrossRef]

16. Rivas, M. Generalized Lagrangians and spinning particles. Ukr. Math. J. 2001, 53, 1326-1339. [CrossRef] 
17. Dirac, P.A.M. The Theory of Gravitation in Hamiltonian Form. Proc. Roy. Soc. A 1958, 246, 333.

18. Deriglazov, A.; Rizzuti, B.F. Reparametrization-invariant formulation of classical mechanics and the Schrödinger equation. Am. J. Phys. 2011, 79, 882-885. [CrossRef]

19. Landau, L.D.; Lifshitz, E.M. The Classical Theory of Fields, 4th ed.; Pergamon Press: Oxford, UK, 1975 ; Volume 2.

20. Dyson, F.J. Feynman's proof of the Maxwell equations. Am. J. Phys. 1990, 58, 209. [CrossRef]

21. Hughes, R.J. On Feynman's proof of the Maxwell equations. Am. J. Phys. 1992, 60, 301. [CrossRef]

22. Randers, G. On an Asymmetrical Metric in the Four-Space of General Relativity. Phys. Rev. 1941, 59, 195-199. [CrossRef]

23. Bouvier, P.; Maeder, A. Consistency of Weyl's Geometry as a Framework for Gravitation. ApESS 1978, 54, 497-508.

24. Maeder, A.; Bouvier, P. Scale invariance, metrical connection and the motions of astronomical bodies. Astron. Astrophys. 1979, 73, 82-89.

25. Hojman, S.; Harleston, H. Equivalent Lagrangians: Multidimensional case. J. Math. Phys. 1981, 22, 1414. [CrossRef]

26. Valentino, E.D.; Anchordoqui, L.A.; Akarsu, O.; Ali-Haimoud, Y.; Amendola, L.; Arendse, N.; Asgari, M.; Ballardini, M.; Basilakos, S.; Battistelli, E.; et al. Cosmology Intertwined I: Perspectives for the Next Decade. arXiv 2020, arXiv:2008.11283.

27. Maeder, A.; Gueorguiev, V.G. The growth of the density fluctuations in the scale-invariant vacuum theory. Phys. Dark Univ. 2019, 25, 100315. [CrossRef]

28. Maeder, A.; Gueorguiev, V.G. The scale-invariant vacuum (SIV) theory: A possible origin of dark matter and dark energy. Universe 2020, 6, 46. [CrossRef]

29. Maeder, A.; Gueorguiev, V.G. Scale-invariant dynamics of galaxies, MOND, dark matter, and the dwarf spheroidals. MNRAS 2019, 492, 2698. [CrossRef]

30. Maeder, A.; Gueorguiev, V.G. Scale Invariance, Horizons, and Inflation. MNRAS 2021, under review.

31. Rucker, R.B. Geometry, Relativity and the Fourth Dimension; Dover: New York, NY, USA, 1977.

32. Magueijo, J.; Smolin, L. Lorentz Invariance with an Invariant Energy Scale. Phys. Rev. Lett. 2002, 88, 190403. [CrossRef]

33. Kleinert, H. Path Collapse In Feynman Formula- Stable Path Integral Formula From Local Time Reparametrization Invariant Amplitude. Phys. Lett. 1989, B224, 313. [CrossRef]

34. Dirac, P.A.M. Generalized Hamiltonian Dynamics. Proc. Roy. Soc. A 1958, 246, 326. [CrossRef]

35. Teitelboim, C. Quantum mechanics of the gravitational field. Phys. Rev. D 1982, 25, 3159. [CrossRef]

36. Henneaux, M.; Teitelboim, C. Quantization of Gauge Systems; Princeton University: Princeton, NJ, USA, 1992.

37. Sundermeyer, K. Constrained Dynamics; Springer: New York, NY, USA, 1982.

38. Gueorguiev, V.G. Matter, Fields, and Reparametrization-Invariant Systems. In Proceedings of the 4th Conference on Geometry, Integrability and Quantization, Varna, Bulgaria, 6-15 June 2002.

39. Gueorguiev, V.G. The Relativistic Particle and Its d-Brane Cousins; Conference Proceedings of Gravity, Astrophysics, and Strings; Fiziev, P.P., Todorov, M.D., Eds.; St. Kliment Ohridski University Press: Sofia, Bulgaria, 2003.

40. Gueorguiev, V.G. Reparametrization-Invariance and Some of the Key Properties of Physical Systems. arXiv 2019, arXiv:1903.02483.

41. Todorov, I.T. Concerning the quantization of a mechanical system with second-order constraints. Ann. Inst. Poincare 1978, A28, 207.

42. Horwitz, L.P.; Rohrlich, F. Constraint relativistic quantum dynamics. Phys. Rev. D 1981, 24, 1528. [CrossRef]

43. Dirac, P.A.M. The Large Numbers Hypothesis and the Einstein Theory of Gravitation. Proc. Roy. Soc. A 1979, $365,19$.

44. Bekenstein, J.D. Relation between physical and gravitational geometry. Phys. Rev. D 1993, 48, 3641. [CrossRef] [PubMed]

45. Goenner, H.F.M. On the History of Geometrization of Space-time: From Minkowski to Finsler Geometry. arXiv 2008, arXiv:0811.4529.

46. Pfeifer, C. Finsler spacetime geometry in physics. Int. J. Geom. Methods Mod. Phys. 2019, 16, 1941004. [CrossRef]

47. Bogoslovsky, G. Some physical displays of the space anisotropy relevant to the feasibility of its being detected at a laboratory. arXiv 2007, arXiv:0706.2621.

48. Bogoslovsky, G.Y. DISIMb(2) Local Relativistic Symmetry and Finslerian Extension of the Theory of Relativity. arXiv 2020, arXiv:2002.02843.

49. Carlip, S. Aberration and the speed of gravity. Phys. Lett. A 2000, 267, 81-87. [CrossRef]

50. Fairlie, D.B.; Ueno, T. Covariant formulation of field theories associated with p-branes. J. Phys. A 2001, 34, 3037. [CrossRef]

51. Pavsic, M. The Landscape of Theoretical Physics; Kluwer Academic Publishers: Boston, MA, USA, 2001.

52. Bozhilov, P. Probe branes dynamics: Exact solutions in general backgrounds. Nucl. Phys. 2003, B656, 199. [CrossRef]

53. Gueorguiev, V.G. Aspects of Diffeomorphism Invariant Theory of Extended Objects. In Quantum Theory and Symmetries, Proceedings of the 3rd International Symposium, Cincinnati, OH, USA, 10-14 September 2003; Argyres, P.C., Hodges, T.J., Mansouri, F., Scanio, J.J., Suranyi, P., Wijewardhana, L.C.R., Eds.; World Scientific: Singapore, 2004.

54. Rohrlich, F. The self-force and radiation reaction. Am. J. Phys. 2000, 68, 1109-1112. [CrossRef]

55. Dirac, P.A.M. Classical Theory of Radiating Electrons. Proc. R. Soc. Lond. Ser. A 1938, 167, 148-169.

56. Polchinski, J. Renormalization and effective lagrangians. Nucl. Phys. B 1984, 231, 269-295. [CrossRef]

57. Adak, M.; Dereli, T.; Ryder, L.H. Neutrino oscillations induced by spacetime torsion. Class. Quantum Grav. 2001, 18, 1503. [CrossRef]

58. Bekaert, X.; Boulanger, N. The unitary representations of the Poincare group in any spacetime dimension. arXiv 2006, arXiv:hepth/0611263. 